\title{
Escherichia coli S2P family intramembrane protease RseP is engaged in the regulated sequential cleavages of FecR in the ferric citrate signaling
}

Tatsuhiko Yokoyama ${ }^{1}$, Tomoya Niinae ${ }^{2}$, Kazuya Tsumagari ${ }^{2}$, Koshi Imami $^{2}$, Yasushi Ishihama ${ }^{2}$, Yohei Hizukuri ${ }^{1, *}$, Yoshinori Akiyama ${ }^{1, *}$

1, Institute for Frontier Life and Medical Sciences, Kyoto University, Kyoto 606-8507, Japan

2, Graduate School of Pharmaceutical Sciences, Kyoto University, Kyoto 606-8501, Japan

*For correspondence:

yakiyama@infront.kyoto-u.ac.jp

yhizukur@infront.kyoto-u.ac.jp

Tel. +81 75-751-4040; Fax +81 75-771-5699

\section{Running title:}

E. coli RseP-catalyzed intramembrane proteolysis of FecR

\section{Keywords:}

metalloprotease, proteomics, metal homeostasis, bacterial signal transduction, siderophore, site- $\underline{2}$ protease (S2P), regulated intramembrane proteolysis (RIP), intramembrane-cleaving proteases (I-CLiPs), iron uptake, multi-step processing 


\begin{abstract}
Escherichia coli RseP, a member of the S2P family of intramembrane proteases, is involved in the activation of the $\sigma^{\mathrm{E}}$ extracytoplasmic stress response and elimination of remnant signal peptides. However, whether RseP has additional cellular functions is unclear. In this study, we attempted to identify new RseP substrates to explore still unknown physiological roles of this protease. Our mass spectrometry-based quantitative proteomic analysis revealed that the levels of several Fec system proteins encoded by the $f e c A B C D E$ operon ( $f e c$ operon) were significantly decreased in an RseP-deficient strain. The Fec system is responsible for the uptake of ferric citrate, and the transcription of the $f e c$ operon is controlled by FecI, an alternative sigma factor, and its regulator FecR, a single-pass transmembrane protein. Assays with the fec operon expression reporter demonstrated that the proteolytic activity of RseP is essential for the ferric citratedependent upregulation of the $f e c$ operon. Analysis using the FecR protein and FecR-derived model proteins showed that FecR undergoes sequential processing at the membrane and that RseP participates in the last step of this sequential processing to generate the $\mathrm{N}$-terminal cytoplasmic fragment of FecR that participates in the transcription of the $f e c$ operon with FecI. Ferric citrate signal-dependent generation of this cleavage product is the essential and sufficient role of RseP in the transcriptional activation of the fec operon. Our study unveiled that E. coli RseP performs the intramembrane proteolysis of FecR, a novel physiological role that is essential for regulating iron uptake by the ferric citrate transport system.
\end{abstract}




\section{Introduction}

While bacterial cellular membranes act as a barrier to protect a cell from extrinsic damages caused by various xenobiotics and hazardous changes in environmental conditions, they must mediate not only selective import of nutrients and other small molecules but also transduction of signals from the external milieu to adapt to environmental changes. A variety of mechanisms exist to transmit information across the membrane. Among them, regulated intramembrane proteolysis (RIP) is a crucial mechanism conserved among all kingdoms $(1,2)$. In RIP, a class of membrane proteases called intramembrane proteases (IMPs) mediate transmembrane signaling through the cleavage of target membrane proteins. IMPs are unique membrane-integrated proteases in that they have their proteolytic active site located within the lipid bilayer and catalyze proteolysis in the

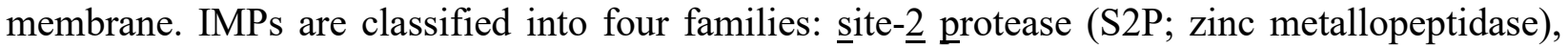
rhomboid protease (serine protease), presenilin/signal peptide peptidase (SPP; aspartyl protease), and Rcel (glutamyl protease) $(3,4)$. IMPs cleave various substrates and thereby play diverse cellular roles including stress responses, development of Alzheimer's disease, induction of apoptosis, maintenance of mitochondrial homeostasis, invasion of apicomplexan parasites, and quality control of membrane proteins and bacterial pathogenicity (5-8).

Escherichia coli RseP is one of the most well-studied members of the S2P family proteases. RseP was first identified as a key factor that regulates the $\sigma^{\mathrm{E}}$ extracytoplasmic stress response through the cleavage of a single membrane-spanning anti- $\sigma^{\mathrm{E}}$ protein, RseA (9-11). In the $\sigma^{\mathrm{E}}$ extracytoplasmic stress response, the accumulation of misfolded outer membrane proteins and lipopolysaccharide biosynthesis intermediates in the cell envelope acts as stress cues to induce the cleavage of RseA by DegS, a membrane-anchored serine protease, on the periplasmic side (site1 cleavage). This first cleavage triggers the following RseP-catalyzed second cleavage of RseA 
inside the membrane (site-2 cleavage), leading to liberation from the membrane of the RseA cytoplasmic domain fragment complexed with $\sigma^{\mathrm{E}}$. Finally, degradation of the RseA cytoplasmic domain fragment by cytoplasmic proteases such as ClpXP activates $\sigma^{\mathrm{E}}$ to induce the transcription of stress genes $(12,13)$. The first cleavage of RseA by DegS is a prerequisite for the subsequent site-2 cleavage by RseP. Similarly, most of the other S2P family proteases are known to catalyze intramembrane proteolysis of a target protein only after preceding trimming of the substrate on the extracytoplasmic side by other protease(s). E. coli RseP has also been shown to eliminate remnant signal peptides generated during the membrane translocation of presecretory proteins that would contribute to the quality control of the cytoplasmic membrane (14). RseP homologs (or S2P family proteases) of many gram-negative and gram-positive bacteria are involved in various cellular processes (7). They include the production of sex pheromones in Enterococcus faecalis (15), sporulation in Bacillus subtilis (16), acid response in Salmonella enterica (17), mucoid conversion and alginate overproduction in Pseudomonas aeruginosa (18), production of cholera toxin in Vibrio cholerae (19), and iron acquisition in Bordetella bronchiseptica and P. aeruginosa $(20,21)$,

RseP spans the membrane 4 times with both of its $\mathrm{N}$ - and C-termini facing the periplasmic space. The residues of the first and third transmembrane segments constitute the intramembrane proteolytic active site $(22,23)$, and the central periplasmic region between the second and the third transmembrane segments contains tandemly-arranged two PDZ domains (PDZ tandem) $(24,25)$ and an amphiphilic helix that is presumably involved in the proper positioning of the PDZ tandem and a substrate (26). RseP and other S2P proteases generally cleave a single-spanning membrane protein with type II ( $\mathrm{N}_{\mathrm{IN}}$-COUT) topology. We previously proposed a model that the bulky PDZ tandem acts as a size-exclusion filter to prevent the access of a substrate with a large periplasmic domain to the protease active site in the membrane $(25,27)$. According to this model, the DegScleaved form of RseA that has lost most of its periplasmic domain (leaving a $\sim 30$ a. a. C-terminal 
periplasmic tail), but not the full-length RseA, can pass through the PDZ filter and access the active site of RseP. Cleavage of other known substrates (remnant signal peptides (14) and a small membrane protein, YqfG (28)) can also be explained by this model, because the formers are generated by the preceding cleavage of precursor secretory proteins around the periplasmic surface of the cytoplasmic membrane by a leader peptidase and this processing is required for the cleavage of signal peptides by RseP, whereas the latter intrinsically has a very small ( $\sim 12$ a. a.) periplasmic domain. The transmembrane segments of the known substrates of RseP share no detectable homology in their primary sequences, suggesting that RseP does not recognize a specific sequence motif(s) in the transmembrane segment of a substrate for its cleavage. Mutational analysis of the transmembrane segments of model substrates showed that the stability of their helical structures is an important determinant for their susceptibility to $\operatorname{RseP}(28,29)$. Consistently, RseP homologs in other bacteria cleave a variety of membrane proteins with no apparent sequence homology. The sequence diversity of the RseP/S2P substrates makes it difficult to predict potential substrates from a simple sequence analysis of transmembrane sequences of membrane proteins. It would thus be reasonable to assume that RseP still has unidentified substrates and plays important roles in some cellular processes by cleaving these substrates and a more comprehensive approach is needed to search for novel substrates and cellular functions of RseP.

In this study, we employed a proteomic approach to achieve the above objective. Proteomic analysis is a powerful technique to identify novel substrates of proteases as reported previously (30-33). We found that the accumulation of several proteins encoded in the $f e c A B C D E$ operon ( $f e c$ operon) decreased considerably in the RseP-deficient strain. The Fec proteins constitute the Fec system that mediates the uptake of ferric citrate by a cell $(34,35)$. We demonstrated that FecR receives sequential processing at the membrane and that RseP plays an essential role in the activation of FecI, an alternative sigma factor dedicated to the transcription of 
the fec operon (36-39), through the regulated intramembrane cleavage of a periplasmically processed form of FecR, the regulator of the fec operon expression (40-42), which produces the N-terminal cytoplasmic tail fragment of FecR having a "pro-sigma" activity (40, 43) as the last step in the sequential processing of FecR at the membrane. These results uncover the details of the FecR processing in the ferric citrate-induced transmembrane signaling and a new physiological role of RseP in the iron uptake in response to the environmental conditions in E. coli.

\section{Results}

\section{Search for new physiological substrates of RseP by quantitative proteomic analysis}

For comprehensive understanding of the cellular functions of RseP in E. coli, we attempted to identify new physiological substrates of RseP by a quantitative proteomic approach. We expected that this analysis would enable us to find not only direct proteolytic substrates of RseP but also proteins whose expression is regulated as a result of substrate proteolysis by RseP. We compared the protein levels in the membrane fractions prepared from the $\Delta$ rse $A$ rseP mutant strain expressing the wild type or a proteolytically inactive form (a mutant having a Glu-23 to Gln alteration (E23Q) in the conserved zinc metallopeptidase active site motif, $\mathrm{HE}^{23} \mathrm{xxH}$ ) of RseP from a plasmid. Although the rseP gene is essential for growth, it can be deleted in the absence of the functional $r s e A$ gene encoding anti- $\sigma^{\mathrm{E}}$ protein RseA, a physiological substrate of RseP. In both the strains, $\sigma^{\mathrm{E}}$ is constitutively activated because of the absence of RseA and thus we can exclude the possible changes in protein expression resulting from RseP-dependent induction of the $\sigma^{\mathrm{E}}$ stress response. The membrane fractions were prepared from these strains grown to mid-log phase in LB broth containing $1 \mathrm{mM}$ IPTG, an inducer of the plasmid-encoded RseP derivatives, and subject to 
nanoLC/MS/MS analysis. As a result, we identified 13,815 unique peptides derived from 1,419 proteins (E. coli strain K-12). Among the identified proteins, 17 exhibited a significant $(P<0.05)$ increase whereas 41 exhibited a significant decrease in the samples prepared from the RseP(E23Q)expressing strain compared with the samples prepared from RseP(WT)-expressing strain (Table 1). Gene ontology enrichment analysis using the DAVID $(44,45)$ showed that the former included groups related to TonB box receptor and transmembrane beta strand, and the latter included proteins related to iron transport (Fold Enrichment $>10$, Table S1). Among the former proteins, three [CyoE (heme O synthase $(46,47)$ ), AmtB (ammonium transporter (48)), and ZupT (heavy metal divalent cation transporter $(49,50))$ ], exhibited a fold change greater than 2 (Fig. 1). Although these proteins accumulated under the RseP-deficient condition, they are unlikely to be the direct substrates of RseP, as S2P proteases generally cleave a single membrane-spanning protein with type II membrane topology. The top three of the latter group were FecA, FecD, and FecE, which also exhibited a fold change greater than 2 . These proteins constitute the Fec system, one of the iron uptake systems in E. coli. Expression of these proteins is known to be induced in response to the availability of environmental iron. The decrease in their levels in the RseP-deficient strain indicates that they should also not be the direct substrates of RseP. However, because it has been shown that many bacterial S2P proteases are involved in cellular responses to environmental changes including the availability of iron $(20,21,51)$, we further examined the possible involvement of RseP in the regulation of the Fec system gene expression.

\section{RseP function is required for the transcriptional activation of the $f e c A B C D E$ operon.}

Since iron is generally an essential micronutrient for living organisms, cells now have many evolved iron-uptake systems (52). An E. coli cell is also equipped with multiple iron uptake systems. Among them, the Fec system acts in the uptake of ferric ion $\left(\mathrm{Fe}^{3+}\right)$ in the form of ferric 
citrate (Fig. 2A). Ferric citrate in the extracellular milieu is first transported into the periplasmic space by FecA, an outer membrane transporter (53-55), and then imported into the cytoplasm via the $\mathrm{ABC}$ transporter FecCDE with the assistance of the periplasmic protein FecB (56). These Fec proteins are expressed from the single $f e c A B C D E$ operon ( $f e c$ operon) whose transcription is under the control of an alternative sigma factor, FecI (36-39). Because the genes for FecA, FecD, and FecE that exhibited decreased accumulation in the RseP-deficient strain belong to the same fec operon (38), we supposed that RseP is involved in the upregulation of this operon, and examined this possibility.

To easily monitor the transcription of the fec operon, we constructed a reporter ( $f e c$ reporter) plasmid in which the lacZ reporter gene was placed under the FecI-dependent promoter of the $f e c$ operon $\left(\mathrm{P}_{\text {fecA }}\right)$. The $\triangle o m p A \Delta o m p C$ cells (used as the $r s e P^{+}$strain) carrying the reporter plasmid exhibited about 10-fold higher LacZ activity when $1 \mathrm{mM} \mathrm{Na}$ citrate was added to the medium containing $0.1 \mu \mathrm{M} \mathrm{FeCl}$ (Fig. 2B, rseP $P^{+}$. Note that the addition of increasing concentrations of $\mathrm{FeCl}_{3}$ to the medium led to a drastic decrease in LacZ activity in both $\triangle o m p A \Delta o m p C$ (Fig. S1A) and wild-type $\left(o m p A^{+} o m p C^{+}\right)$cells (Fig. S1B). This effect was presumably caused by the negative regulation of $\mathrm{P}_{\text {fec }}$ by Fur, a global transcriptional regulator for iron homeostasis $(38,39,41,57)$. These results showed that our reporter plasmid can be used to evaluate the transcription from $\mathrm{P}_{\text {fec }}$ in response to ferric citrate.

We then introduced the reporter plasmid into the $\triangle o m p A \Delta o m p C \Delta r s e P$ cells $(r s e P$ can be deleted in a strain lacking the two outer membrane proteins OmpA and OmpC (58)) and examined the effect of $r s e P$ disruption on the transcriptional activation of the fec operon in response to ferric citrate. In sharp contrast to $r \mathrm{seP}^{+}$cells, the expression of the fec operon as revealed by LacZ activity was not increased by the addition of citrate (Fig. 2B, $\Delta r s e P /$ vec). Expression of the wild-type RseP with a C-terminal His6-Myc tag (hereafter RseP-HM), but not its E23Q derivative, from 
another plasmid restored a citrate-dependent reporter expression (Fig. 2B, WT and E23Q), while the anti-RseP immunoblotting showed that the accumulation levels of the expressed RseP proteins were comparable (Fig. 2C). These results strongly suggest that the proteolytic activity of RseP is essential for the ferric citrate-dependent transcriptional activation of the fec operon.

\section{Proteolytic function of RseP is involved in the processing of the FecR protein.}

The transcription of the $f e c$ operon is known to be controlled by FecI, an alternative sigma factor whose activity is regulated by FecR (40-42) (Fig. 2A). FecR is a single-spanning cytoplasmic membrane protein of type II topology, similar to other RseP substrates, and has been reported to be processed into several fragments in vivo $(59,60)$. In addition, its $\mathrm{N}$-terminal cytoplasmic region has been suggested to exhibit an activity ("pro-sigma" activity) that is required for the function of FecI $(40,43)$. These facts led us to examine the possibility that FecR is cleaved by RseP to activate FecI.

We first investigated whether RseP could affect the in vivo processing of FecR. To this end, we constructed a derivative of FecR with an N-terminal 3xFLAG tag (F-FecR) (Fig. 3A). We also constructed a new $\Delta f e c R(\Delta f e c R l)$ strain in which a part of the chromosomal $f e c R$ gene was deleted so that the deletion totally disrupts the FecR function but negligibly affects the expression of the upstream and downstream genes (see Supplementary Experimental Procedures). Assays with the $f e c$ reporter using the $\Delta f e c R$ cells showed that the expression of F-FecR restored the ferric citrate-dependent induction of transcription from $\mathrm{P}_{f e c A}$ that was abolished in the $\Delta f e c R$ cells, indicating that F-FecR is functional (Fig. 3B). We examined the effects of rseP deletion on the processing of the FecR protein (Fig. 3C). F-FecR was expressed in a $\Delta r s e A$ rse $P^{+}$and a $\Delta r s e A \Delta r s e P$ strain by growing the cells in M9 medium supplemented with $1 \mathrm{mM} \mathrm{Na}$-citrate and $10 \mu \mathrm{M} \mathrm{FeCl}_{3}$ (Fig. 3C, lanes 9 and 10 ). Note that $10 \mu \mathrm{M} \mathrm{FeCl}_{3}$ was added to the medium during 
the analyses of the FecR processing to obtain clear and reproducible results (see Supplementary Results). Anti-FLAG immunoblotting with total cellular proteins showed that in addition to the full-length F-FecR band ( 37 kDa; FL), several smaller FecR-derived fragments were accumulated; FL and an $\sim 25 \mathrm{kDa}$ band (labeled as CL(a)) were detected in both $r s e P^{+}$and $\Delta r s e P$ strains, whereas a $\sim 17 \mathrm{kDa}$ band (CL(b)) and a $\sim 15 \mathrm{kDa}$ band (CL(c)) were detected only in $r s e P^{+}$ and $\Delta$ rseP strains, respectively. Also, CL(b) and CL(c) were generated in a citrate-dependent manner (Fig. 3C, compare lanes 5 and 6 with 9 and 10). While CL(a) was generated irrespective of the addition of citrate, the accumulation levels of CL(a) were considerably higher in the absence of citrate than in its presence (Fig. 3C, compare lanes 5 and 6 with 9 and 10). Taken together, these results suggest that ferric citrate affects the generation and/or accumulation of FecR species in the cells. All these species (CL(a), CL(b) and CL(c)) should be N-terminal fragments of F-FecR as they retained the N-terminal FLAG tag, and most likely are the degradation products of FecR. Citrate did not affect the accumulation levels of the RseP proteins in these strains. When wild-type RseP-HM was ectopically expressed in the $\Delta r s e P$ stain, CL(b) disappeared and instead CL(c) was detected, while no such effect was observed with the expression of the protease active-site mutant RseP(E23Q) (Fig. 3C, lanes 11 and 12). These results suggest that CL(c) is generated by the RsePdependent proteolytic cleavage of the FecR-related proteins.

\section{RseP cleaves the transmembrane region of FecR to yield the cytoplasmic tail fragment.}

In the above immunoblotting experiments, we observed significant variations in the relative amounts of the FecR-related species (data not shown). We suspected that these variations could be caused by the instability of these species in a cell. In addition, the positive feedback regulation of the Fec system $(34,35)$ could complicate the results as it could enhance the ferric citrate-dependent signal and as a result the signal-induced processing of FecR. To circumvent these 
problems, we constructed a FecR-derived model substrate, F-MBP-FecR, in which the entire cytoplasmic domain of FecR was replaced with a tightly folded MBP (maltose binding protein) domain (Fig. 4A). This approach has proved to be useful to analyze the RseP-mediated or periplasmic proteolysis in our past studies $(14,29)$. The expression of F-MBP-FecR in the $\Delta f e c R$ strain did not activate the expression of the $f e c$ reporter in the presence or absence of citrate, suggesting that it is not functional, as expected from its lack of the cytoplasmic domain required for the interaction with and activation of FecI (Fig. 3B). Next, we examined the in vivo processing of the F-MBP-FecR protein by immunoblotting (Fig. 4B and S2). In the presence of citrate, we obtained essentially the same results as those obtained with F-FecR; we detected four F-MBPFecR-derived species that should correspond to FL, CL(a), CL(b) and CL(c), ascertaining from their sizes, and CL(c) was generated in the presence of chromosomal or ectopically expressed functional RseP, whereas CL(b) was detected instead of CL(c) under RseP-deficient conditions. Without the addition of citrate, the amounts of CL(a) were increased substantially both in the presence and absence of functional RseP, which also reproduced the results with F-FecR (Fig. 4B, compare lanes 5-8 with 9-12). Remarkably, even in the absence of citrate, small but significant amounts of the CL(b) and CL(c) bands were detected with F-MBP-FecR and the pattern of their generation was essentially the same as that in the presence of citrate, whereas we did not observe CL(b) and CL(c) with F-FecR in the absence of citrate. Quantification of the band intensities demonstrated high reproducibility of the results with F-MBP-FecR (Fig. S2). These observations suggest that (i) F-MBP-FecR retains its "responsibility" to ferric citrate with respect to the generation of the N-terminal fragments, although it is not functional in signal transduction and no longer enhances the Fec signaling through its positive feedback loop, and (ii) the replacement of the cytoplasmic domain of FecR with the F-MBP domain stabilizes the N-terminal fragments, which would allow for the detection of CL(b) and CL(c) without the addition of ferric citrate. These 
results demonstrate that F-MBP-FecR can be used as a suitable model substrate for studying of the FecR processing.

To estimate the approximate position of the C-terminal end of the CL(a), CL(b), and CL(c) fragments, we constructed a series of C-terminally truncated versions of F-MBP-FecR (Fig. 4A). The transmembrane region of FecR was predicted to be located between residues 80 and 100 (with the N-terminal Met residue of FecR set to residue 1) by the topology prediction algorithm SPOCTOPUS (http://octopus.cbr.su.se/index.php)(61). Accordingly, we constructed two truncated mutants, F-MBP-FecR101 and F-MBP-FecR79, in which the regions after residues 101 and 79 had been deleted, respectively. F-MBP-FecR79 lacks the predicted transmembrane and periplasmic regions, whereas F-MBP-FecR101 only lacks the periplasmic region. Because an alternative region (residues 86 to 100) of FecR has been suggested to span the membrane in a previous study (42), we also constructed a truncated mutant (F-MBP-FecR85) that lacked the region after residue 85 . In addition, based on the previous studies that reported the self-cleavage of FecR between Gly-181 and Thr-182 (59, 62, 63), we constructed F-MBP-FecR181 without the region after residue 181, as a mimic of the N-terminal fragment generated by self-cleavage. Finally, we systematically constructed three additional C-terminally truncated mutants (F-MBP-FecR161, 141, and 121) by deleting C-terminal 20, 40, or 60 residues from F-MBP-FecR181, respectively. These constructs were expressed in $\Delta r s e A \Delta r s e P$ cells and their sizes were compared with the CL(a), CL(b) and CL(c) fragments that had been generated from full-length F-MBP-FecR in vivo (Fig. 4C). With FMBP-FecR181, two bands having nearly the same mobilities as CL(a) and CL(b), respectively, were detected (Fig. 4C, compare lanes 1-3 with 4). The former band would be intact in F-MBPFecR181, suggesting that CL(a) is a self-cleavage product of F-MBP-FecR $(59,62,63)$. Similarly, two bands were observed with F-MBP-FecR161, 141, and 121 (Fig. 4C, lanes 4-7), and the upper bands would also represent their "intact" form. The second (smaller) bands generated for the above 
four constructs had almost the same apparent size as CL(b). In contrast, only a single band was detected for F-MBP-FecR101, 85, and 79 (Fig. 4C, lanes 8-10), each having no periplasmic region. Taken together, these results strongly suggest that FecR receives a cleavage in its periplasmic region to generate $\mathrm{CL}(\mathrm{b})$. As the "intact" band of F-MBP-FecR121 was slightly larger than the CL(b) band, the possible cleavage site would be located just upstream of reside 121 .

The mobility of CL(c), whose production is dependent on the proteolytic function of RseP, was almost the same as that of F-MBP-FecR85 (compare Fig. 4C, lanes 8-10 and 11), suggesting that CL(c) would be generated by the RseP-mediated cleavage around residue 85 . This cleavage site is highly likely to be located within the transmembrane region of FecR, given that RseP cleaves a transmembrane segment of a substrate. Accordingly, our results support the prediction that the FecR spans the membrane in the region between residues 80 and 100 .

\section{RseP cleaves the FecR CL(b) fragment and converts it to the CL(c) fragment}

The apparent RseP-dependent conversion of CL(b) to CL(c) can be well-explained if CL(c) is produced by the intramembrane proteolysis of CL(b) by RseP. To substantiate this possibility, the stability and the production kinetics of F-MBP-FecR and its derivatives (CL(a), CL(b) and CL(c)) were investigated by pulse-chase experiments (Fig. 5 and S3). F-MBP-FecR was expressed in the $\Delta r s e P$ strain, labeled with $\left[{ }^{35} \mathrm{~S}\right]$ methionine and chased with unlabeled methionine up to 81 min after initiation of the chase. FL and CL(a) band were the only species detected just after the start of the chase (Fig. 5A, and the close-up view of the quantified results for the early periods of the pulse-chase experiments is shown in Fig. S3B, at 0.25 -min time point). FL almost completely disappeared within 3-min chase. On the other hand, CL(a) decreased gradually with time with a small amount detectable even after the 81 min-chase (Fig. 5B). In contrast, CL(b) appeared at 1-min chase point and increased during the chase period. Co-expression of wild-type 
RseP-HM only marginally accelerated the disappearance of FL and CL(a). In this case, however, CL(b) was not detected, but instead CL(c) was generated. CL(c) increased with a similar kinetics to that of CL(b) observed in the absence of the RseP-HM co-expression. Generation of CL(c) was dependent on the proteolytic function of RseP-HM as co-expression of RseP(E23Q)-HM did not exert such an effect, although the accumulation levels of RseP-HM and RseP(E23Q)-HM were comparable (Fig. S3A). These results strongly suggest that both CL(b) and CL(c) are derived from CL(a). However, it was still unclear from these results whether CL(c) was generated from CL(a) or generated from $\mathrm{CL}(\mathrm{b})$.

To address this point, we designed an assay procedure (Figs. 6A, 6B and S4) in which cells expressing F-MBP-FecR from a constitutive expression vector were pulsed-labeled and chased for $5.5 \mathrm{~min}$, and then, RseP-HM or RseP(E23Q)-HM was induced (Fig. 6A, B). At 0.5 min before the induction of RseP, only CL(a) and CL(b) were detected. We expected that, if RseP is indeed involved in the cleavage of CL(b), (i) this experimental system could enable us to detect the possible RseP-dependent conversion of pre-existing CL(b) to CL(c), and also (ii) the presence of an excess amount of the unlabeled FL and CL(c) molecules (as the result of their constitutive expression) compared with RseP-HM that could retard apparent degradation rate of labeled CL(b) and facilitate detection of the conversion process. Actually, we captured the CL(b) to CL(c) conversion upon the induction of RseP-HM but not that of RseP(E23Q)-HM. These results strongly support the idea that RseP directly cleaves CL(b) to produce CL(c). Although the accumulation levels of RseP-HM and RseP(E23Q)-HM varied significantly in the two independent experiments (Fig. S4B), the results of the pulse-chase analysis of the F-MBP-FecR derivatives were highly reproducible (Fig. 6B and Fig. S4A). Although the reason of this observation remains unclear, it might suggest that only a limited portion of overexpressed RseP acts in the CL(b) cleavage. 
To confirm the direct involvement of RseP in cleavage of CL(b), we examined the effect of RseP on the degradation of the C-terminally truncated mutants of F-MBP-FecR. F-MBPFecR121, which lacked almost the entire periplasmic region, had a slightly larger size than that of CL(b) and generated CL(b) but not CL(a). The model proteins were co-expressed with wild-type or the E23Q mutant form of RseP-HM (Figs. 4A and 6C). CL(b) generated from F-MBP-FecR121 and F-MBP-FecR181 were converted to CL(c) in the functional RseP-dependent manner. (Fig. 6C, lanes 4-7) On the other hand, the band pattern and accumulation of F-MBP-FecR85, which mimics the CL(c) fragment, was little affected by the RseP function (Fig. 6C, lanes 8 and 9). These results further support the RseP-catalyzed cleavage of CL(b) to yield CL(c).

\section{The FecR CL(b) fragment is generated from the CL(a) fragment}

CL(b) was generated from F-MBP-FecR181 that mimics CL(a) (Fig. 4C lane 4 and Fig. 6C lane 5). It would be thus conceivable that $\mathrm{CL}(\mathrm{b})$ is a degradation product of CL(a). Our pulsechase results, showing the apparent precursor-product relationship between CL(a) and CL(b) (that is, the disappearance of CL(a) is accompanied by the appearance of CL(b)) (Fig. 5), also strongly support that CL(a) is converted to CL(b). Immunoblotting analysis showed that the addition of citrate to the medium led to the increase in the accumulation of CL(b) and the concomitant decrease in that of CL(a) (Compare Fig. 3C lanes 6 with 10 (F-FecR), or Fig. 4B lanes 6 with 10 (F-MBPFecR; the quantified results of Fig. 4B are shown in Fig. S2). These results suggest that the CL(a) to $\mathrm{CL}(\mathrm{b})$ conversion is promoted by the ferric citrate signal.

The essential function of RseP in the transcriptional activation of the fec operon is to produce the FecR CL(c) fragment 
The above results showed that RseP proteolytically converts FecR CL(b) to CL(c). The generation of CL(c) was well-correlated with the ferric citrate-dependent activation of the $f e c$ operon (Fig. 2 and 3). In addition, a previous study showed that the expression of the predicted cytoplasmic domain of FecR (FecR(1-85)) (40, 42, 64), which mimics CL(c), activated the transcription of the fec operon with no need of the ferric citrate signal. These results strongly suggest that RseP activates the $f e c$ operon by producing the $\mathrm{CL}(\mathrm{c})$ fragment. However, it still cannot be excluded that RseP plays an additional role in the activation of the fec operon. We thus investigated the requirement of RseP in the $f e c$ operon activation by the cytoplasmic fragment of FecR (Fig.7). When expressed in the $\Delta f e c R$ strain, F-FecR85, a 3xFLAG-tagged cytoplasmic domain (residue 1 to 85) of FecR showed almost the same mobility as that of CL(c) generated from F-FecR (Fig. 7A, compare lanes 5 and 6 with 4 ), further supporting that CL(c) is generated by the cleavage around the residue 85. F-FecR85 accumulated at similar levels in the presence or absence of citrate (Fig. 7A, lanes 5 and 6). Also, the expression of F-FecR85, in contrast to that of full-length F-FecR, activated the $f e c$ operon transcription in a citrate-independent manner (Fig. 7B, pF-FecR). The Nterminally attached 3xFLAG tag little affected the function of FecR and FecR85 as the un-tagged versions of these proteins gave essentially the same results (Fig. 7B, compare pFecR with pF-FecR). LacZ activities showed apparent correlations with the accumulation levels of F-FecR85 or F-FecR CL(c) fragment (Fig. 7A, lanes 3-6 and Fig. 7B, pF-FecR). These results confirmed the previous finding that the cytoplasmic domain of FecR can constitutively activate the transcription of the fec operon $(40,42,64)$. The RseP function was not required for this ferric citrate-independent activation of the $f e c$ operon (Fig. 7C), as the reporter activities of the cells expressing F-FecR85 were elevated considerably in a citrate-independent manner both in the presence or absence of chromosomal rseP. Consistent with the above result, the accumulation level of F-FecR85 in the $r s e P^{+}$and $\Delta r s e P$ cells was not affected by the addition of citrate (Fig. S5). In this experiment, the 
reporter activities in the $\Delta r s e P$ cells were apparently a little higher than those in the $r s e P^{+}$cells, possibly reflecting the increased accumulation of the F-FecR85 protein in the former cells (Fig. S5), although the exact reason for this differential accumulation is unclear. Taken together, these results demonstrated that the essential role of $\mathrm{RseP}$ in the activation of the fec operon is to produce the CL(c) fragment that co-functions with FecI from CL(b).

\section{Discussion}

In this study, we performed proteomic analysis to identify the substrates of RseP, the S2P family intramembrane protease of $E$. coli, to explore novel functions of this protease, and unveiled that RseP is involved in the regulation of the Fec system (Ferric citrate uptake system) genes through the intramembrane cleavage of a novel physiological substrate, FecR. Our proteomic analysis identified multiple Fec system components (FecA, FecD and FecE) encoded by the fec operon as proteins whose levels were significantly decreased in the RseP-deficient strain, suggesting that $\mathrm{RseP}$ is required for the expression of the $f e c$ operon. We thus examined the transcriptional regulation of this operon by using a lacZ-reporter and demonstrated that proteolytic activity of RseP is essential for its activation. Transcription of this operon is known to be controlled by FecI, an alternative sigma factor (36-39), whose activity is regulated by a cytoplasmic membrane protein, FecR (40-42). Since FecR is a single-pass transmembrane protein of type II topology, which is a shared feature of substrates of the bacterial S2P proteases including RseP, we examined the possibility that RseP cleaves this protein to induce expression of the fec operon. The experiments using the FecR-derived model substrates revealed that FecR receives sequential processing at the membrane and that RseP participates in the last step in the processing that 
generates the cytoplasmic tail fragment of FecR required for the transcriptional activation of the fec operon.

FecR was processed to yield the fragments that we named here CL(a), CL(b), and CL(c) (Fig. 5). We demonstrated that RseP cleaves the transmembrane region of CL(b), and converts it to CL(c) (Fig. 6). The production of CL(c) is required and sufficient for the FecI-mediated activation of the $f e c$ operon transcription (Fig. 2 and 7). These observations coincide with and further support the results of previous studies, which showed that the expression of the cytoplasmic region of FecR led to the ferric citrate-independent activation of the transcription of the $f e c$ operon $(40,42,64)$. A previous study showed that the N-terminal cytoplasmic region of FecR can directly interact with FecI (60) and acts as a "pro-sigma" factor to facilitate the transcription of the fec operon. It is thus suggested that the RseP-mediated cleavage of CL(b) would liberate CL(c) from the membrane to activate the $f e c$ operon transcription by forming a complex with FecI. Braun et al. (2006) previously proposed a model wherein the RseP-catalyzed intramembrane proteolysis of FecR is involved in the activation of the $f e c$ operon (65), although it has not been demonstrated experimentally. Our present study provided the strong evidence supporting this model. RseP orthologues in gramnegative bacteria act in processing of sigma factor regulators to activate cognate sigma factors in a variety of iron acquiring systems (e.g., B. bronchiseptica HurR (21), P. aeruginosa FpvR (20), FoxR (63), HxuR, HasS (66) and P. putida IutY (51)). Our study added another example to this list.

Our results suggest that $\mathrm{CL}(\mathrm{b})$ is produced by the cleavage of CL(a) and that this process is promoted by the ferric citrate signal. Thus, CL(a) apparently receives two successive cleavages to yield CL(c), which is reminiscent of the two-step cleavage of E. coli RseA triggered by the extracytoplasmic stresses. In case of RseA, it is recognized and cleaved by RseP (“site-2 cleavage") only after it received prior "site-1 cleavage" by the membrane serine protease DegS on the periplasmic side. While the two successive cleavages are common among many bacterial S2P 
substrates, the protease catalyzing the site-1 cleavage is not necessarily a DegS homolog. For example, B. subtilis PrsW, a multipass metalloprotease that cleaves RsiW in response to antimicrobial peptides and envelope stresses is unrelated to E. coli DegS (67). Also, in case of $P$. putida IutY, periplasmic serine protease Prc is possibly responsible for its "site-1 cleavage" (51). Although we have no information on the putative protease(s) responsible for the production of CL(b) form CL(a) in the FecR-mediated signal transduction, the ferric citrate signal might induce some conformational change in CL(a) to make it susceptible to the site-1 cleavage. Alternatively, the ferric citrate signal might directly activate the putative "site-1" protease. Further analysis, especially the identification of the putative "site-1" protease, will be needed to reveal the detailed molecular mechanism of this process.

The production of CL(a) occurred immediately after the synthesis of FecR and independently of RseP. A previous study suggested that CL(a) is generated by the cleavage between Gly-181 and Thr-182 (59), which is consistent with our result that CL(a) migrated on an SDSPAGE gel to almost the same position as the FecR-derivative that had been truncated at Gly-181. This GT motif is conserved among several anti-sigma factors of P. aeruginosa and P. putida (63) and E. coli FecR. Furthermore, Bastiaansen et al. (2015) showed that one of them, P. aeruginosa FoxR, underwent self-cleavage at this site and suggested that the other GT motif containing antisigma proteins are also processed auto-catalytically at this motif in the non-enzymatic fashion, i.e., $\mathrm{N}-\mathrm{O}$ acyl rearrangement (62). It would be very likely that E. coli FecR CL(a) is generated by the self-cleavage at the GT motif, too. Whether prior autoproteolysis is required for the production of CL(b) is unclear. Previous studies showed that some mutations in the GT motif of FoxR, which receives a cleavage by RseP during the signal transduction like FecR, blocks the self-cleavage but still allow iron-dependent signal transduction, suggesting that the self-cleavage is not essential in this system, although it cannot be excluded that the FoxR mutants with the GT motif mutations 
receive degradation by some proteases to generate a small amount of a CL(a)-like fragment, which is sufficient to drive the down-stream reactions. If the self-cleavage of FecR is not required for the signal transduction in the E. coli Fec system as suggested for FoxR, the possible protease responsible for generation of CL(b) might be able to cleave both of the FL and CL(a) forms of FecR to provide a substrate of RseP, as RseP should not cleave full-length FecR like RseA discussed below.

We propose a model of the FecR-processing cascade in the signal transduction by the Fec system (Fig. 8) based on the results of preceding studies by other researchers and the present study. (i) FecR is self-cleaved almost immediately after synthesis and generates CL(a). (ii) In response to the ferric citrate signal transmitted via FecA, CL(a) receives the $\mathrm{C}$-terminal truncation or trimming by unknown protease(s) in the periplasmic space to generate CL(b). (iii) CL(b) is then recognized and cleaved by RseP within the membrane, leading to the release of CL(c) from the membrane. (iv) Finally, the liberated CL(c) that is associated with the FecI promotes the transcription of the fec operon. The approaches used in this study, especially detailed pulse-chase experiments and analyses with various model proteins, will be useful to understand the details of the processing of other proteins including $P$. aeruginosa FoxR for which similar multi-step cleavages have been proposed but their timings and relationships are not fully understood. Regulated intramembrane proteolysis (RIP) catalyzed by intramembrane proteases is widely found in many organisms from bacteria to eukaryote $(1,2)$. The cleavage of FecR to induce the fec operon transcription in the signal-dependent activation of the Fec system would be one of the typical instances of RIPmediated transmembrane signaling.

We previously proposed that the periplasmic PDZ tandem of RseP serves as a sizeexclusion filter to avoid proteolysis of membrane proteins with a large periplasmic domain by RseP (27). In the $\sigma^{\mathrm{E}}$ extracytoplasmic stress response, the site-1 protease DegS is activated by the stress 
signals and cleaves full-length RseA, the ant $-\sigma^{\mathrm{E}}$ protein, in its periplasmic region, which triggers the following site-2 cleavage by RseP as the RseA degradation intermediate (RseA148) generated by the site- 1 cleavage has a small periplasmic region $(\sim 30$ a. a.) and can get access to the intramembrane active site passing through the PDZ filter. Thus, the PDZ filter ensures the stress dependent activation of $\sigma^{\mathrm{E}}$ (Fig. S6) $(68,69)$. Similarly, in the case of FecR we expect that the conversion of CL(b), which is supposed to have a periplasmic region of about 80 a. a., to CL(c) with a smaller periplasmic tail ( $\sim 20$ a. a.) would make FecR accessible to the intramembrane active site of RseP through the PDZ filter. As the CL(b)-to-CL(c) conversion is promoted by the ferric citrate, the PDZ filter-mediated discrimination of the substrates contributes to the ferric citrate signal-dependent activation of FecI as well.

The predicted transmembrane sequence of FecR $\left(\mathrm{R}^{80} \mathrm{HVMKGLLLLLGAGGGWQLWQ}^{100}\right.$ ) has no apparent sequence homology to the known substrates of RseP $(14,28,29)$. We previously suggested that the stability of a transmembrane helix in the membrane, but not the exact primary sequence, is an important determinant for its susceptibility to cleavage by $\operatorname{RseP}(28,29)$. Consistent with this notion, RseA and FecR have several residues such as Lys, Arg, and Gln that potentially destabilize a helix in a hydrophobic environment (70) in their predicted transmembrane segments. These residues might be required for the efficient cleavage of these proteins by RseP. In addition, similar to RseA, the transmembrane sequence of FecR has relatively low hydrophobicity compared with transmembrane segments of typical membrane proteins such as lactose permease (LacY). The assigned transmembrane sequences of FecR and RseA are not predicted as a transmembrane segments by a transmembrane prediction program such as TMHMM (71) in contrast to the transmembrane segments of LacY (Fig. S7). The products of intramembrane proteolysis could be released from the membrane because they have a shorter hydrophobic stretch and the polarity or charges at their N- and C- 
termini generated by the intramembrane cleavage (72). The low hydrophobicity of the transmembrane segments of RseA and FecR could further facilitate efficient liberation of the Nterminal fragments of RseA and FecR from the membrane after the RseP-mediated intramembrane proteolysis and accelerate the transmembrane signaling in each system. Interestingly, the first and fifth transmembrane segments of LacY can be efficiently cleaved by RseP when placed in a model substrate (29) despite of their high hydrophobicity, suggesting that the overall hydrophobicity of a transmembrane segment is not a critical determinant for its susceptibility to RseP.

Proteomic analysis is a powerful approach that allows non-biased search for protease substrates. Thus far, many examples have been reported in which proteomic analysis was successfully used to identify the substrate of membrane proteases including E. coli FtsH (32), B. subtilis MgtE (30), Homo sapiens PARL (33), etc. We thus employed this approach and identified FecR as novel substrate of RseP. In this analysis we found significant changes in the accumulation levels of some proteins, in addition to the Fec proteins, depending on the proteolytic activity of RseP. Among the group of proteins that exhibited increased accumulation in the RseP-deficient strain, CyoE (73), Fiu (74), CirA (75), and FepA (76) have been reported to be negatively regulated by the Fur protein. Fur acts as a global repressor for the expression of genes involved in iron uptake upon its interaction with iron (77). The increased expression of the Fur-regulated genes in the cells expressing RseP(E23Q) might at least partially be ascribed to a decreased intracellular iron level resulting from the lowered expression of the Fec components. In contrast to the above-mentioned proteins, the increased accumulation was observed for AmtB and ZupT (fold change $>2$ ) in the cells expressing RseP(E23Q) (Fig. 1 and Table. 1). Both AmtB and ZupT are transporters of the cytoplasmic membrane; the former facilitates the uptake of ammonia $(48,78)$ whereas the latter imports divalent metal cations $(49,50)$. Since these proteins are multi-spanning transmembrane proteins, it is unlikely that RseP directly proteolyze these proteins. Their expression or stability 
might be indirectly affected by the loss of the proteolytic function of RseP. It should be noted that FecR evaded detection in our proteomic analyses. One possibility would be that the FecR level was below the detection threshold in our experiments. Another possibility would be that most of the FecR molecules were lost during the preparation of the membrane fraction. As discussed above, FecR and RseA have a transmembrane segment of relatively low hydrophobicity. We often encountered the significant decrease or loss of RseA and its derivatives in the membrane fraction possibly as a result of their falling-out from the membrane (unpublished observations). It is possible that we overlooked other RseP substrates by similar and/or other reasons. For the systematic identification of RseP substrates, it is necessary to improve the methods of sample preparations in mass analyses $(31,79,80)$ and also use additional approaches such as the co-isolation of possible substrates with a proteolytically inactive RseP and systematic screening of type II membrane proteins in combination with the mass analysis-based proteomic approach. Such approaches would also be useful to search for the unknown substrate of other IMPs.

Recently, the Fec system has been reported to be crucial in bovine mastitis caused by a pathogenic E. coli strain (81). Bovine mastitis is a disease in dairy cows that causes economic loss to the global dairy industry. This study and further analysis of the regulation of the Fec system may hopefully contribute to unveiling the mechanism and development of the treatment of this disease. Our mass analysis results suggest that the levels of the proteins involved in a variety of cellular activities such as ion transport, rRNA processing, and acetylation are affected by the impairment of the RseP function (Table S1). It raises the possibility that RseP may have additional substrates and act directly or indirectly in still unknown cellular processes. Further study by using a variety of approaches discussed above will lead to the comprehensive understanding of the significance of RseP in cellular activities. 


\section{Experimental procedures}

\section{Media}

L broth (10 g/L Bacto Tryptone, $5 \mathrm{~g} / \mathrm{L}$ yeast extract and $5 \mathrm{~g} / \mathrm{L} \mathrm{NaCl}$; $\mathrm{pH}$ adjusted to 7.2 by using $\mathrm{NaOH}$ ) and M9 medium (without $\mathrm{CaCl}_{2}$ ) (82) supplemented with $2 \mu \mathrm{g} / \mathrm{mL}$ thiamine and $0.4 \%$ glucose were used for cultivation of $E$. coli cells. Ampicillin $(50 \mu \mathrm{g} / \mathrm{mL})$, chloramphenicol $(20 \mu \mathrm{g} / \mathrm{mL})$ and/or spectinomycin $(50 \mu \mathrm{g} / \mathrm{mL})$ were added for selecting transformants and for growing plasmid-harboring cells. Bacterial growth was monitored using mini photo 518R (660 nm; TAITEC) or Klett-Summerson colorimeter (filter no. 54; Klett Manufacturing).

\section{Strains, plasmids and oligonucleotides}

Escherichia coli $\mathrm{K}-12$ strains, plasmids and oligonucleotides used in this work are listed in Supplementary Tables S2, S3 and S4, respectively. Construction of the individual strains and plasmids are described in Supplementary Experimental Procedures.

\section{Antibodies}

Monoclonal anti-FLAG M2 antibody (MilliporeSigma), Rabbit polyclonal anti-RseP antibody (83) and anti-SecB antibody (26) were used for immunoblotting. Monoclonal anti-FLAG M2 Affinity Gel (MilliporeSigma) was used for Immunoprecipitation in pulse-chase assay.

\section{NanoLC/MS/MS sample preparation}

Cells were grown in L broth supplemented with $0.4 \%$ glucose, $1 \mathrm{mM}$ IPTG and $1 \mathrm{mM}$ cAMP at $30^{\circ} \mathrm{C}$ until a mid-log phase. Harvested cells were washed with $10 \mathrm{mM}$ Tris- $\mathrm{HCl}(\mathrm{pH} 8.1)$ and suspended in buffer containing $10 \mathrm{mM}$ Tris- $\mathrm{HCl}(\mathrm{pH} \mathrm{8.1)}$ and protease Inhibitor cocktail 
(Nacalai Tesque). Cell suspension was frozen, thawed, and lysed by sonication. After cell debris was removed by low speed centrifugation, membrane fractions were collected by ultracentrifugation (at 125,000 x $g, 60 \mathrm{~min}$ ) and suspended in PTS solution (12 mM Sodium deoxycholate, $12 \mathrm{mM}$ sodium N-lauroylsarcosinate, $100 \mathrm{mM}$ Tris-HCl (pH 9.0)) (84). After incubation at room temperature for $30 \mathrm{~min}$ and at $37^{\circ} \mathrm{C}$ for $5 \mathrm{~min}$, the protein amount was confirmed with a BCA protein assay kit (Thermo Fisher Scientific). Proteins were reduced by treatment with $10 \mathrm{mM}$ DTT for $30 \mathrm{~min}$, alkylated by treatment with $50 \mathrm{mM}$ chloroacetamide for $30 \mathrm{~min}$ in the dark. Next, the protein solution was 5-fold diluted with $50 \mathrm{mM}$ ammonium bicarbonate and the proteins were digested with Lys-C (w/w 1:100) for $3 \mathrm{~h}$, followed by trypsin digestion (w/w 1:100) overnight at $37^{\circ} \mathrm{C}$. The peptides were desalted using StageTip (85) with SDB-XC Empore disk membranes (GL Sciences). The peptides were labeled with Tandem Mass Tag (TMT) (Thermo Fisher Scientific), fractionated using strong cation exchange chromatography (SCX) StageTips (86) and suspended in the loading solution (0.5\% TFA and 4\% ACN) for subsequent nanoLC/MS/MS analyses.

\section{NanoLC/MS/MS analyses}

NanoLC/MS/MS analyses were performed on an Orbitrap Fusion Lumos (Thermo Fisher Scientific), connected to an Ultimate 3000 pump (Thermo Fisher Scientific) and an HTC-PAL autosampler (CTC Analytics). Peptides were separated on a self-pulled needle column (150 mm length $\times 100 \mu \mathrm{m}$ ID, $6 \mu \mathrm{m}$ opening) packed with Reprosil-C18 AQ $3 \mu \mathrm{m}$ reversed-phase material (Dr. Maisch). The flow rate was set to $500 \mathrm{~nL} / \mathrm{min}$. The mobile phase consisted of (A) $0.5 \%$ acetic acid and (B) $0.5 \%$ acetic acid in $80 \%$ acetonitrile. Three-step linear gradients of $5-10 \%$ B in 5 min, $10-40 \% \mathrm{~B}$ in $60 \mathrm{~min}$, and $40-100 \% \mathrm{~B}$ in $5 \mathrm{~min}$ were employed. For TMT-labeled samples, synchronous precursor selection-MS3 (SPS-MS3) (87) was performed. The MS scan range was 
$m / z$ 375-1500. MS scans were performed by the Orbitrap with $r=120,000$, MS/MS scans were performed by the Ion Trap in Turbo mode and MS3 scans were performed by the Orbitrap with $r=$ 15,000. Auto gain control was set to $4.00 \times 10^{5}, 1.00 \times 10^{4}, 5.00 \times 10^{4}$ for MS, MS/MS and MS3, respectively. The normalized CID collision energy was set to 35 .

\section{Database searching}

The raw MS data files were analyzed by MaxQuant v1.6.2.3 (88). Peptides and proteins were identified by means of automated database searching using Andromeda against the E. coli K12 SwissProt Database (version 2018-07, 4,324 protein entries) with a precursor mass tolerance of $20 \mathrm{ppm}$ for first search and $4.5 \mathrm{ppm}$ for main search and a fragment ion mass tolerance of $0.5 \mathrm{Da}$ Enzyme was set as trypsin/P (cleaves after lysine and arginine also if a proline follows) and semispecific search was performed. Cysteine carbamidomethylation was set as a fixed modification. Methionine oxidation and acetylation on protein N-termini were set as variable modifications. The search results were filtered with FDR $<1 \%$ at the peptide spectrum match (PSM) and protein levels.

\section{$\beta$-galactosidase (LacZ) activity assay}

LacZ activity of the cells carrying the reporter plasmid pYK149 $\left(\mathrm{P}_{\text {fecA }}-l a c Z\right)$ was measured basically according to the procedure described previously (89). The cells were grown at $30^{\circ} \mathrm{C}$ in M9-based medium with $20 \mu \mathrm{g} / \mathrm{mL}$ each of the 20 amino acids, $2 \mathrm{mg} / \mathrm{mL}$ thiamine and $0.4 \%$ glucose until mid-log phase. The cells were mixed with Reporter 5xLysis buffer (Promega), frozen at $-80^{\circ} \mathrm{C}$ for more than $1 \mathrm{~h}$, and thawed by incubation at $37^{\circ} \mathrm{C}$ for $30 \mathrm{~min}$ in a clear 96 -well plate. Then, the equal volume of Z-buffer $\left(60 \mathrm{mM} \mathrm{Na} \mathrm{HPO}_{4} \cdot 7 \mathrm{H}_{2} \mathrm{O}, 40 \mathrm{mM} \mathrm{NaH} \mathrm{PO}_{4} \cdot \mathrm{H}_{2} \mathrm{O}, 10 \mathrm{mM} \mathrm{KCl}, 1 \mathrm{mM}\right.$ $\mathrm{MgSO}_{4} \cdot 7 \mathrm{H}_{2} \mathrm{O}, \quad 40 \mathrm{mM} \quad \beta$-mercaptoethanol) containing $1.32 \mathrm{mg} / \mathrm{mL} \quad 2$-nitrophenyl $\beta$-Dgalactopyranoside (ONPG, MilliporeSigma) was added to this lysate and incubated at room 
temperature, followed by detection of the absorbance at 420 and $550 \mathrm{~nm}$ at every $2 \mathrm{~min}$ using the Viento Nano microplate reader (BioTek Instruments). The relative LacZ activity was calculated as follows. First, the "raw LacZ activity" was calculated according to the following equation; "raw LacZ activity (arbitrary units)" $=\left(\mathrm{A}_{420}-1.75 \mathrm{x} \mathrm{A}_{550}\right) /($ incubation time $(\mathrm{min}))$. The value of the raw LacZ activity of each sample was divided by that of a standard cell sample (CU141 cells cultured in M9-based medium), and then by $\mathrm{A}_{600}$ of the bacterial culture at the time of collection, giving the "corrected LacZ activity". Finally, the relative LacZ activity of each sample was obtained by dividing the value of the corrected LacZ activity by that of the corresponding control (see the legends for the control in each experiment).

\section{SDS-PAGE}

Proteins were dissolved in SDS sample buffer (62.5 mM Tris-HCl ( $\mathrm{pH} \mathrm{8.5),2 \%} \mathrm{(w/v)}$ SDS, $10 \%$ glycerol, $10 \% \beta$-mercaptoethanol, trace of bromophenol blue) and separated by SDSPAGE using $10 \%$ or $12.5 \%$ Laemmli gel as described previously (90). For separation of the F-FecR protein and its derivatives, SDS-PAGE using a 15\% Bis-Tris gel (107 mM bis-Tris (pH6.8 with $\mathrm{HCl}$ ), 15\% Acrylamide/Bis, 0.1\% APS, 0.1\% TEMED) and MES SDS running buffer (50 mM Tris, $50 \mathrm{mM}$ MES, $1 \mathrm{mM}$ EDTA, $0.1 \%(\mathrm{w} / \mathrm{v})$ SDS) was used.

\section{Immunoblotting}

Cells were grown at $30^{\circ} \mathrm{C}$ in M9-based medium with $20 \mu \mathrm{g} / \mathrm{mL}$ each of the 20 amino acids, $2 \mu \mathrm{g} / \mathrm{mL}$ thiamine and $0.4 \%$ glucose until mid-log phase. Total cellular proteins were precipitated with 5\% trichloroacetic acid (TCA), washed with acetone and dissolved in SDS sample buffer. Immunoblotting was carried out essentially as described previously $(90,91)$. Proteins were separated by SDS-PAGE and electroblotted onto an Immobilon-P membrane filter 
(MilliporeSigma). Only when 15\% bis-Tris gel was used for SDS-PAGE, a transferred membrane filter was dried at $37^{\circ} \mathrm{C}$ for $30 \mathrm{~min}$ and then hydrophilized with methanol. After blocking with BLOTTO (90), the filter was incubated with an appropriate antibody. For anti-RseP immunoblotting, anti-RseP antibodies were pre-incubated with whole-cell lysates of AD1840 (the $\Delta r s e A \Delta r s e P \triangle d e g S$ strain) at $4^{\circ} \mathrm{C}$ for $1 \mathrm{~h}$ to reduce a background as described previously (83). The filter was then washed, and incubated with goat anti-mouse or anti-rabbit IgG conjugated with horseradish peroxide (Bio-Rad). After washing of the filter, proteins that reacted with secondary antibodies were visualized using ECL or ECL Prime Western Blotting Detection Reagents (Cytiva) and Bio image analyzer LAS4000mini (Cytiva).

\section{Pulse-chase experiment}

Cells were grown at $30^{\circ} \mathrm{C}$ in M9-based medium with $20 \mu \mathrm{g} / \mathrm{mL}$ each of the 18 amino acids (other than methionine and cysteine), $2 \mu \mathrm{g} / \mathrm{mL}$ thiamine and $0.4 \%$ glucose until early-log phase. In the experiments shown in Fig. 5 and Fig. S3B, the cells were induced with $1 \mathrm{mM}$ IPTG and $1 \mathrm{mM}$ cAMP for $10 \mathrm{~min}$ before pulse-labeling with $\left[{ }^{35} \mathrm{~S}\right]$ methionine for $1 \mathrm{~min}$ and chase with an excess amount of unlabeled methionine for the indicated periods. In the experiments shown in Fig. 6A, B and Fig. S4A, cells were pulse-labelled with $\left[{ }^{35} \mathrm{~S}\right]$ methionine for $1 \mathrm{~min}$ and chased with an excess amount of unlabeled methionine where $1.2 \mathrm{mM}$ IPTG and $1.2 \mathrm{mM}$ cAMP were added to the medium 5.5 min after the initiation of the chase. The proteins were precipitated with 5\% TCA, washed with acetone and dissolved in $30 \mu \mathrm{L}$ of $50 \mathrm{mM}$ Tris- $\mathrm{HCl}(\mathrm{pH} 8.1)$ containing $1 \mathrm{mM}$ EDTA and $1 \%$ SDS by vigorous mixing for $30 \mathrm{~min}$ at room temperature followed by incubation at $37^{\circ} \mathrm{C}$ for $5 \mathrm{~min}$. The samples were diluted 33-fold with $1 \mathrm{~mL}$ Triton buffer (50 mM Tris-HCl (pH 8.1), $0.1 \mathrm{mM}$ EDTA, 2\% Triton $\mathrm{X}-100,150 \mathrm{mM} \mathrm{NaCl}$ ). After clarification by centrifugation, the supernatants were mixed with anti-FLAG M2 Affinity Gel and Protein A Sepharose (Cytiva) and 
incubated at $4{ }^{\circ} \mathrm{C}$ overnight. The antigen-antibody complexes were recovered by centrifugation, washed with Triton buffer and then with $10 \mathrm{mM}$ Tris- $\mathrm{HCl}(\mathrm{pH} 8.1)$ and dissolved in SDS sample buffer. The proteins were separated by SDS-PAGE and visualized and quantified using a phosphor imager (BAS5000) (Cytiva).

\section{Data availability}

The MS raw data and analysis files have been deposited to the ProteomeXchange Consortium (http://proteomecentral.proteomexchange.org) via the jPOST partner repository (http://jpostdb.org) (92) with the data set identifier PXD023797.

\section{Acknowledgments}

We thank Kazue Kanehara for constructing the plasmid pKK47. We thank Hiroyuki Mori,

Ryoji Miyazaki, Eiji Ishii, and all the other lab members for stimulating discussion, and Michiyo Sano and Satomi Koshiba for secretarial support, and National BioResource Project (NBRP) $-E$. coli at National Institute of Genetics, Japan for strains. We also thank Editage for English language editing.

\section{Conflict of interest}

The authors declare that they have no conflicts of interest with the contents of this article. 


\section{References}

1. Brown, M. S., Ye, J., Rawson, R. B., and Goldstein, J. L. (2000) Regulated intramembrane proteolysis: a control mechanism conserved from bacteria to humans. Cell. 100, 391-8

2. Wolfe, M. S. (2009) Intramembrane proteolysis. Chem. Rev. 109, 1599-1612

3. Sun, L., Li, X., and Shi, Y. (2016) Structural biology of intramembrane proteases: Mechanistic insights from rhomboid and S2P to $\gamma$-secretase. Curr. Opin. Struct. Biol. 37, $97-107$

4. Beard, H. A., Barniol-Xicota, M., Yang, J., and Verhelst, S. H. L. (2019) Discovery of Cellular Roles of Intramembrane Proteases. ACS Chem. Biol. 14, 2372-2388

5. Deu, E. (2017) Proteases as antimalarial targets: strategies for genetic, chemical, and therapeutic validation. FEBS J. 284, 2604-2628

6. Kühnle, N., Dederer, V., and Lemberg, M. K. (2019) Intramembrane proteolysis at a glance: from signalling to protein degradation. J. Cell Sci. 132, jcs217745

7. Schneider, J. S., and Glickman, M. S. (2013) Function of site-2 proteases in bacteria and bacterial pathogens. Biochim. Biophys. Acta. 1828, 2808-2814

8. Konovalova, A., Søgaard-Andersen, L., and Kroos, L. (2014) Regulated proteolysis in bacterial development. FEMS Microbiol. Rev. 38, 493-522

9. Kanehara, K., Ito, K., and Akiyama, Y. (2002) YaeL (EcfE) activates the $\sigma^{\mathrm{E}}$ pathway of stress response through a site-2 cleavage of anti- $\sigma^{\mathrm{E}}$, RseA. Genes Dev. 16, 2147-2155

10. Kanehara, K., Akiyama, Y., and Ito, K. (2001) Characterization of the yaeL gene product and its S2P-protease motifs in Escherichia coli. Gene. 281, 71-79 
11. Alba, B. M., Leeds, J. A., Onufryk, C., Lu, C. Z., and Gross, C. A. (2002) DegS and YaeL participate sequentially in the cleavage of RseA to activate the $\sigma^{\mathrm{E}}$-dependent extracytoplasmic stress response. Genes Dev. 16, 2156-2168

12. Kroos, L., and Akiyama, Y. (2013) Biochemical and structural insights into intramembrane metalloprotease mechanisms. Biochim. Biophys. Acta. 1828, 2873-2885

13. Ades, S. E. (2008) Regulation by destruction: design of the $\sigma^{\mathrm{E}}$ envelope stress response. Curr. Opin. Microbiol. 11, 535-540

14. Saito, A., Hizukuri, Y., Matsuo, E. I., Chiba, S., Mori, H., Nishimura, O., Ito, K., and Akiyama, Y. (2011) Post-liberation cleavage of signal peptides is catalyzed by the site-2 protease (S2P) in bacteria. Proc. Natl. Acad. Sci. U. S. A. 108, 13740-13745

15. Dunny, G. M. (2013) Enterococcal Sex Pheromones: Signaling, Social Behavior, and Evolution. Annu. Rev. Genet. 47, 457-482

16. Yu, Y. T. N., and Kroos, L. (2000) Evidence that SpoIVFB is a novel type of membrane metalloprotease governing intercompartmental communication during Bacillus subtilis sporulation. J. Bacteriol. 182, 3305-3309

17. Muller, C., Bang, I. S., Velayudhan, J., Karlinsey, J., Papenfort, K., Vogel, J., and Fang, F. C. (2009) Acid stress activation of the $\sigma^{\mathrm{E}}$ stress response in Salmonella enterica serovar Typhimurium. Mol. Microbiol. 71, 1228-1238

18. Delgado, C., Florez, L., Lollett, I., Lopez, C., Kangeyan, S., Kumari, H., Stylianou, M., Smiddy, R. J., Schneper, L., Sautter, R. T., Smith, D., Szatmari, G., and Mathee, K. (2018) Pseudomonas aeruginosa regulated intramembrane proteolysis: Protease MucP can overcome mutations in the $\mathrm{AlgO}$ periplasmic protease to restore alginate production in nonmucoid revertants. J. Bacteriol. 200, e00215-18 
19. Pennetzdorfer, N., Lembke, M., Pressler, K., Matson, J. S., Reidl, J., and Schild, S. (2019) Regulated Proteolysis in Vibrio cholerae Allowing Rapid Adaptation to Stress Conditions. Front. Cell. Infect. Microbiol. 9, 214

20. Draper, R. C., Martin, L. W., Beare, P. A., and Lamont, I. L. (2011) Differential proteolysis of sigma regulators controls cell-surface signalling in Pseudomonas aeruginosa. Mol. Microbiol. 82, 1444-1453

21. King-Lyons, N. D., Smith, K. F., and Connell, T. D. (2007) Expression of hurP, a gene encoding a prospective site 2 protease, is essential for heme-dependent induction of bhuR in Bordetella bronchiseptica. J. Bacteriol. 189, 6266-6275

22. Feng, L., Yan, H., Wu, Z., Yan, N., Wang, Z., Jeffrey, P. D., and Shi, Y. (2007) Structure of a Site-2 Protease Family Intramembrane Metalloprotease. Science. 318, 1608-1612

23. Koide, K., Maegawa, S., Ito, K., and Akiyama, Y. (2007) Environment of the active site region of RseP, an Escherichia coli regulated intramembrane proteolysis protease, assessed by site-directed cysteine alkylation. J. Biol. Chem. 282, 4553-4560

24. Li, X., Wang, B., Feng, L., Kang, H., Qi, Y., Wang, J., and Shi, Y. (2009) Cleavage of RseA by RseP requires a carboxyl-terminal hydrophobic amino acid following DegS cleavage. Proc. Natl. Acad. Sci. U. S. A. 106, 14837-14842

25. Inaba, K., Suzuki, M., Maegawa, K. I., Akiyama, S., Ito, K., and Akiyama, Y. (2008) A pair of circularly permutated PDZ domains control RseP, the S2P family intramembrane protease of Escherichia coli. J. Biol. Chem. 283, 35042-35052

26. Miyake, T., Hizukuri, Y., and Akiyama, Y. (2020) Involvement of a Membrane-Bound Amphiphilic Helix in Substrate Discrimination and Binding by an Escherichia coli S2P Peptidase RseP. Front. Microbiol. 11, 607381 
27. Hizukuri, Y., Oda, T., Tabata, S., Tamura-Kawakami, K., Oi, R., Sato, M., Takagi, J., Akiyama, Y., and Nogi, T. (2014) A Structure-Based Model of Substrate Discrimination by a Noncanonical PDZ Tandem in the Intramembrane-Cleaving Protease RseP. Structure. 22, $326-336$

28. Akiyama, K., Mizuno, S., Hizukuri, Y., Mori, H., Nogi, T., and Akiyama, Y. (2015) Roles of the membrane-reentrant $\beta$-hairpin-like loop of RseP protease in selective substrate cleavage. eLife. 4, e08928

29. Akiyama, Y., Kanehara, K., and Ito, K. (2004) RseP (YaeL), an Escherichia coli RIP protease, cleaves transmembrane sequences. EMBO J. 23, 4434-4442

30. Began, J., Cordier, B., Březinová, J., Delisle, J., Hexnerová, R., Srb, P., Rampírová, P., Kožíšek, M., Baudet, M., Couté, Y., Galinier, A., Veverka, V., Doan, T., and Strisovsky, K. (2020) Rhomboid intramembrane protease YqgP licenses bacterial membrane protein quality control as adaptor of FtsH AAA protease. EMBO J. 39, e102935

31. Tsumagari, K., Shirakabe, K., Ogura, M., Sato, F., Ishihama, Y., and Sehara-Fujisawa, A. (2017) Secretome analysis to elucidate metalloprotease-dependent ectodomain shedding of glycoproteins during neuronal differentiation. Genes to Cells. 22, 237-244

32. Arends, J., Thomanek, N., Kuhlmann, K., Marcus, K., and Narberhaus, F. (2016) In vivo trapping of FtsH substrates by label-free quantitative proteomics. Proteomics. 16, 31613172

33. Saita, S., Nolte, H., Fiedler, K. U., Kashkar, H., Saskia, A. V., Zahedi, R. P., Krüger, M., and Langer, T. (2017) PARL mediates Smac proteolytic maturation in mitochondria to promote apoptosis. Nat. Cell Biol. 19, 318-328

34. Braun, V., Mahren, S., and Ogierman, M. (2003) Regulation of the Fecl-type ECF sigma factor by transmembrane signalling. Curr. Opin. Microbiol. 6, 173-180 
35. Braun, V., and Mahren, S. (2005) Transmembrane transcriptional control (surface signalling) of the Escherichia coli Fec type. FEMS Microbiol. Rev. 29, 673-684

36. Härle, C., Kim, I., Angerer, A., and Braun, V. (1995) Signal transfer through three compartments: transcription initiation of the Escherichia coli ferric citrate transport system from the cell surface. EMBO J. 14, 1430-1438

37. Angerer, A., Enz, S., Ochs, M., and Braun, V. (1995) Transcriptional regulation of ferric citrate transport in Escherichia coli K-12. Fecl belongs to a new subfamily of $\sigma^{70}$-type factors that respond to extracytoplasmic stimuli. Mol. Microbiol. 18, 163-174

38. Enz, S., Braun, V., and Crosa, J. H. (1995) Transcription of the region encoding the ferric dicitrate-transport system in Escherichia coli: similarity between promoters for fecA and for extracytoplasmic function sigma factors. Gene. 163, 13-18

39. Ochs, M., Angerer, A., Enz, S., and Braun, V. (1996) Surface signaling in transcriptional regulation of the ferric citrate transport system of Escherichia coli: Mutational analysis of the alternative sigma factor FecI supports its essential role in $f e c$ transport gene transcription. Mol. Gen. Genet. 250, 455-465

40. Ochs, M., Veitinger, S., Kim, I., Weiz, D., Angerer, A., and Braun, V. (1995) Regulation of citrate-dependent iron transport of Escherichia coli: FecR is required for transcription activation by Fecl. Mol. Microbiol. 15, 119-132

41. Van Hove, B., Staudenmaier, H., and Braun, V. (1990) Novel two-component transmembrane transcription control: regulation of iron dicitrate transport in Escherichia coli K-12. J. Bacteriol. 172, 6749-6758

42. Welz, D., and Braun, V. (1998) Ferric citrate transport of Escherichia coli: Functional regions of the FecR transmembrane regulatory protein. J. Bacteriol. 180, 2387-2394 
43. Braun, V., and Hantke, K. (2020) Novel Tat-Dependent Protein Secretion. J. Bacteriol. 202, e00058-20

44. Huang, D. W., Sherman, B. T., and Lempicki, R. A. (2009) Bioinformatics enrichment tools: Paths toward the comprehensive functional analysis of large gene lists. Nucleic Acids Res. 37, 1-13

45. Huang, D. W., Sherman, B. T., and Lempicki, R. A. (2009) Systematic and integrative analysis of large gene lists using DAVID bioinformatics resources. Nat. Protoc. 4, 44-57

46. Saiki, K., Mogi, T., and Anraku, Y. (1992) Heme O biosynthesis in Escherichia coli: The cyoE gene in the cytochrome $B O$ operon encodes a protoheme IX farnesyltransferase. Biochem. Biophys. Res. Commun. 189, 1491-1497

47. Saiki, K., Mogi, T., Ogura, K., and Anraku, Y. (1993) In vitro heme O synthesis by the cyoE gene product from Escherichia coli. J. Biol. Chem. 268, 26041-26044

48. Soupene, E., He, L., Yan, D., and Kustu, S. (1998) Ammonia acquisition in enteric bacteria: Physiological role of the ammonium/methylammonium transport B (AmtB) protein. Proc. Natl. Acad. Sci. U. S. A. 95, 7030-7034

49. Grass, G., Wong, M. D., Rosen, B. P., Smith, R. L., and Rensing, C. (2002) ZupT is a Zn(II) uptake system in Escherichia coli. J. Bacteriol. 184, 864-6

50. Grass, G., Franke, S., Taudte, N., Nies, D. H., Kucharski, L. M., Maguire, M. E., and Rensing, C. (2005) The metal permease ZupT from Escherichia coli is a transporter with a broad substrate spectrum. J. Bacteriol. 187, 1604-1611

51. Bastiaansen, K. C., Ibañez, A., Ramos, J. L., Bitter, W., and Llamas, M. A. (2014) The Prc and RseP proteases control bacterial cell-surface signalling activity. Environ. Microbiol. 16, $2433-2443$ 
52. Saha, R., Saha, N., Donofrio, R. S., and Bestervelt, L. L. (2013) Microbial siderophores: A mini review. J. Basic Microbiol. 53, 303-317

53. Wagegg, W., and Braun, V. (1981) Ferric citrate transport in Escherichia coli requires outer membrane receptor protein FecA. J. Bacteriol. 145, 156-163

54. Pressler, U., Staudenmaier, H., Zimmermann, L., and Braun, V. (1988) Genetics of the iron dicitrate transport system of Escherichia coli. J. Bacteriol. 170, 2716-2724

55. Ferguson, A. D., Chakraborty, R., Smith, B. S., Esser, L., Van Der Helm, D., and Deisenhofer, J. (2002) Structural basis of gating by the outer membrane transporter FecA. Science. 295, 1715-1719

56. Staudenmaier, H., Van Hove, B., Yaraghi, Z., and Braun, V. (1989) Nucleotide sequences of the $f e c B C D E$ genes and locations of the proteins suggest a periplasmic-binding-proteindependent transport mechanism for iron(III) dicitrate in Escherichia coli. J. Bacteriol. 171, 2626-2633

57. Angerer, A., and Braun, V. (1998) Iron regulates transcription of the Escherichia coli ferric citrate transport genes directly and through the transcription initiation proteins. Arch. Microbiol. 169, 483-490

58. Douchin, V., Bohn, C., and Bouloc, P. (2006) Down-regulation of porins by a small RNA bypasses the essentiality of the regulated intramembrane proteolysis protease RseP in Escherichia coli. J. Biol. Chem. 281, 12253-12259

59. Wriedt, K., Angerer, A., and Braun, V. (1995) Transcriptional regulation from the cell surface: Conformational changes in the transmembrane protein FecR lead to altered transcription of the ferric citrate transport genes in Escherichia coli. J. Bacteriol. 177, 33203322 
60. Enz, S., Mahren, S., Stroeher, U. H., and Braun, V. (2000) Surface signaling in ferric citrate transport gene induction: Interaction of the FecA, FecR, and FecI regulatory proteins. $J$. Bacteriol. 182, 637-646

61. Viklund, H., Bernsel, A., Skwark, M., and Elofsson, A. (2008) SPOCTOPUS: A combined predictor of signal peptides and membrane protein topology. Bioinformatics. 24, 2928-2929

62. Bastiaansen, K. C., Van Ulsen, P., Wijtmans, M., Bitter, W., and Llamas, M. A. (2015) Selfcleavage of the Pseudomonas aeruginosa cell-surface signaling anti-sigma factor FoxR occurs through an N-O acyl rearrangement. J. Biol. Chem. 290, 12237-12246

63. Bastiaansen, K. C., Otero-Asman, J. R., Luirink, J., Bitter, W., and Llamas, M. A. (2015) Processing of cell-surface signalling anti-sigma factors prior to signal recognition is a conserved autoproteolytic mechanism that produces two functional domains. Environ. Microbiol. 17, 3263-3277

64. Stiefel, A., Mahren, S., Ochs, M., Schindler, P. T., Enz, S., and Braun, V. (2001) Control of the ferric citrate transport system of Escherichia coli: mutations in region 2.1 of the FecI extracytoplasmic-function sigma factor suppress mutations in the FecR transmembrane regulatory protein. J. Bacteriol. $\mathbf{1 8 3}, 162-70$

65. Braun, V., Mahren, S., and Sauter, A. (2006) Gene regulation by transmembrane signaling. BioMetals. 19, 103-113

66. Otero-Asman, J. R., García-García, A. I., Civantos, C., Quesada, J. M., and Llamas, M. A. (2019) Pseudomonas aeruginosa possesses three distinct systems for sensing and using the host molecule haem. Environ. Microbiol. 21, 4629-4647

67. Ellermeier, C. D., and Losick, R. (2006) Evidence for a novel protease governing regulated intramembrane proteolysis and resistance to antimicrobial peptides in Bacillus subtilis. Genes Dev. 20, 1911-1922 
68. Kanehara, K., Ito, K., and Akiyama, Y. (2003) YaeL proteolysis of RseA is controlled by the PDZ domain of YaeL and a Gln-rich region of RseA. EMBO J. 22, 6389-6398

69. Walsh, N. P., Alba, B. M., Bose, B., Gross, C. A., and Sauer, R. T. (2003) OMP peptide signals initiate the envelope-stress response by activating DegS protease via relief of inhibition mediated by its PDZ domain. Cell. 113, 61-71

70. Liu, L. P., and Deber, C. M. (1998) Uncoupling hydrophobicity and helicity in transmembrane segments. $\alpha$-helical propensities of the amino acids in non-polar environments. J. Biol. Chem. 273, 23645-23648

71. Krogh, A., Larsson, B., Von Heijne, G., and Sonnhammer, E. L. L. (2001) Predicting transmembrane protein topology with a hidden Markov model: Application to complete genomes. J. Mol. Biol. 305, 567-580

72. Strisovsky, K. (2016) Why cells need intramembrane proteases - a mechanistic perspective. FEBS J. 283, 1837-1845

73. Stojiljkovic, I., Bäumler, A. J., and Hantke, K. (1994) Fur regulon ingram-negative bacteria: Identification and characterization of new iron-regulated Escherichia coli genes by a Fur titration assay. J. Mol. Biol. 236, 531-545

74. Newman, D. L., and Shapiro, J. A. (1999) Differential fiu-lacZ fusion regulation linked to Escherichia coli colony development. Mol. Microbiol. 33, 18-32

75. Griggs, D. W., and Konisky, J. (1989) Mechanism for iron-regulated transcription of the Escherichia coli cir gene: metal-dependent binding of fur protein to the promoters. $J$. Bacteriol. 171, 1048-1054

76. Hunt, M. D., Pettis, G. S., and McIntosh, M. A. (1994) Promoter and operator determinants for Fur-mediated iron regulation in the bidirectional fepA-fes control region of the Escherichia coli enterobactin gene system. J. Bacteriol. 176, 3944-3955 
77. Andrews, S. C., Robinson, A. K., and Rodríguez-Quiñones, F. (2003) Bacterial iron homeostasis. FEMS Microbiol. Rev. 27, 215-237

78. van Heeswijk, W. C., Westerhoff, H. V., and Boogerd, F. C. (2013) Nitrogen Assimilation in Escherichia coli: Putting Molecular Data into a Systems Perspective. Microbiol. Mol. Biol. Rev. 77, 628-695

79. Bhagwat, S. R., Hajela, K., and Kumar, A. (2018) Proteolysis to Identify Protease Substrates: Cleave to Decipher. Proteomics. 18, 1800011

80. Tsumagari, K., Chang, C.-H., and Ishihama, Y. (2020) Exploring the Landscape of Ectodomain Shedding by Quantitative Protein Terminomics. bioRxiv. $10.1101 / 2020.09 .23 .310102$

81. Blum, S. E., Goldstone, R. J., Connolly, J. P. R., Répérant-Ferter, M., Germon, P., Inglis, N. F., Krifucks, O., Mathur, S., Manson, E., Mclean, K., Rainard, P., Roe, A. J., Leitner, G., and Smith, D. G. E. (2018) Postgenomics characterization of an essential genetic determinant of mammary pathogenic Escherichia coli. mBio. 9, 1-11

82. Miller, J. H. (1972) Experiments in Molecular Genetics, Cold Spring Harbor Laboratory, New York

83. Hizukuri, Y., and Akiyama, Y. (2012) PDZ domains of RseP are not essential for sequential cleavage of RseA or stress-induced $\sigma^{\mathrm{E}}$ activation in vivo. Mol. Microbiol. 86, 1232-1245

84. Masuda, T., Tomita, M., and Ishihama, Y. (2008) Phase transfer surfactant-aided trypsin digestion for membrane proteome analysis. J. Proteome Res. 7, 731-740

85. Rappsilber, J., Mann, M., and Ishihama, Y. (2007) Protocol for micro-purification, enrichment, pre-fractionation and storage of peptides for proteomics using StageTips. Nat. Protoc. 2, 1896-1906 
86. Adachi, J., Hashiguchi, K., Nagano, M., Sato, M., Sato, A., Fukamizu, K., Ishihama, Y., and Tomonaga, T. (2016) Improved proteome and phosphoproteome analysis on a cation exchanger by a combined acid and salt gradient. Anal. Chem. 88, 7899-7903

87. McAlister, G. C., Nusinow, D. P., Jedrychowski, M. P., Wühr, M., Huttlin, E. L., Erickson, B. K., Rad, R., Haas, W., and Gygi, S. P. (2014) MultiNotch MS3 enables accurate, sensitive, and multiplexed detection of differential expression across cancer cell line proteomes. Anal. Chem. 86, 7150-7158

88. Cox, J., and Mann, M. (2008) MaxQuant enables high peptide identification rates, individualized p.p.b.-range mass accuracies and proteome-wide protein quantification. Nat. Biotechnol. 26, 1367-1372

89. Mori, H., Sakashita, S., Ito, J., Ishii, E., and Akiyama, Y. (2018) Identification and characterization of a translation arrest motif in VemP by systematic mutational analysis. $J$. Biol. Chem. 293, 2915-2926

90. Akiyama, K., Hizukuri, Y., and Akiyama, Y. (2017) Involvement of a conserved GFG motif region in substrate binding by RseP, an Escherichia coli S2P protease. Mol. Microbiol. 104, 737-751

91. Hizukuri, Y., Akiyama, K., and Akiyama, Y. (2017) Biochemical Characterization of Function and Structure of RseP, an Escherichia coli S2P Protease. Methods Enzymol. 584, 1-33

92. Moriya, Y., Kawano, S., Okuda, S., Watanabe, Y., Matsumoto, M., Takami, T., Kobayashi, D., Yamanouchi, Y., Araki, N., Yoshizawa, A. C., Tabata, T., Iwasaki, M., Sugiyama, N., Tanaka, S., Goto, S., and Ishihama, Y. (2019) The jPOST environment: an integrated proteomics data repository and database. Nucleic Acids Res. 47, D1218-D1224 


\title{
Funding
}

This work was supported by JSPS KAKENHI Grant Numbers JP18K14674, JP20H03241, JP20H04844 (to KI), JP17H05667 (to YI), JP17K15091, JP19K06562 (to YH), JP18H02404 (to YA) and by JST Strategic Basic Research Program CREST (No. 18070870) to YI.

\begin{abstract}
Abbreviations
The abbreviations used are: FL, full length; IMP, intramembrane protease; IPTG, isopropyl- $\beta$-Dthiogalactopyranoside; MBP, maltose binding protein; TM, transmembrane; WT, wild type.
\end{abstract}




\section{Table}

Table. 1

Significantly changed proteins $(p$-value $<0.05)$ depending on the proteolytic activity of RseP.

\section{UP regulated}

\begin{tabular}{lllll}
$\begin{array}{l}\mathbf{L o g}_{2} \\
\text { (E23Q/WT) }\end{array}$ & $\begin{array}{l}\text {-Log } \mathbf{1 0} \\
\text { p-value }\end{array}$ & $\begin{array}{l}\text { Gene } \\
\text { names }\end{array}$ & Protein names & $\begin{array}{l}\text { Locati } \\
\text { on }\end{array}$ \\
\hline 2.20 & 3.80 & cyoE & Protoheme IX farnesyltransferase & IM \\
1.54 & 2.94 & amtB & Ammonia channel & IM \\
1.14 & 1.55 & zupT & Zinc transporter ZupT & M \\
0.85 & 1.73 & $y c f J$ & Uncharacterized protein YcfJ & M \\
0.63 & 1.67 & fiu & Catecholate siderophore receptor Fiu & OM \\
0.62 & 1.65 & hdeB & Acid stress chaperone HdeB & Peri \\
0.61 & 1.76 & osmB & Osmotically-inducible lipoprotein B & M \\
0.58 & 2.01 & $m d t A$ & Multidrug resistance protein MdtA & IM \\
0.57 & 1.85 & cirA & Colicin I receptor & OM \\
0.55 & 1.41 & wzc & Tyrosine-protein kinase wZc & IM \\
0.52 & 1.38 & glnB & Nitrogen regulatory protein P-II 1 & - \\
0.48 & 1.56 & $y j i Y$ & Inner membrane protein YjiY & IM \\
0.46 & 1.46 & rseP & Regulator of sigma-E protease RseP & IM \\
0.46 & 1.49 & fepA & Ferrienterobactin receptor & OM \\
0.44 & 1.72 & $y q i C$ & Uncharacterized protein YqiC & Cyto \\
0.44 & 1.77 & zntA & Lead, cadmium, zinc and mercury-transporting ATPase & IM \\
0.39 & 2.18 & $m d t B$ & Multidrug resistance protein MdtB & IM
\end{tabular}

\section{DOWN regulated}

\begin{tabular}{|c|c|c|c|c|}
\hline $\begin{array}{l}\log _{2} \\
(\mathrm{E23Q} / \mathrm{WT})\end{array}$ & $\begin{array}{l}-\log _{10} \\
p \text {-value }\end{array}$ & $\begin{array}{l}\text { Gene } \\
\text { names }\end{array}$ & Protein names & $\begin{array}{l}\text { Locati } \\
\text { on }\end{array}$ \\
\hline-3.30 & 4.15 & $f e c D$ & $\mathrm{Fe}(3+)$ dicitrate transport system permease protein $\mathrm{FecD}$ & IM \\
\hline-3.27 & 4.42 & fec $A$ & $\mathrm{Fe}(3+)$ dicitrate transport protein $\mathrm{Fec} \mathrm{A}$ & $\mathrm{OM}$ \\
\hline-1.80 & 3.90 & fecE & $\mathrm{Fe}(3+)$ dicitrate transport ATP-binding protein FecE & $\mathrm{IM}$ \\
\hline-0.76 & 2.06 & yeeR & Inner membrane protein YeeR & IM \\
\hline-0.72 & 1.76 & zпиA & High-affinity zinc uptake system protein ZnuA & Peri \\
\hline-0.71 & 1.39 & $f l u$ & Antigen 43; Antigen 43 alpha chain; Antigen 43 beta chain & Peri \\
\hline-0.69 & 1.47 & sucD & Succinyl-CoA ligase [ADP-forming] subunit alpha & - \\
\hline-0.64 & 1.39 & yaaA & UPF0246 protein YaaA & - \\
\hline-0.58 & 1.34 & putA & $\begin{array}{l}\text { Bifunctional protein PutA; Proline dehydrogenase; Delta-1- } \\
\text { pyrroline-5-carboxylate dehydrogenase }\end{array}$ & - \\
\hline-0.56 & 1.51 & $\operatorname{cstA}$ & Carbon starvation protein $\mathrm{A}$ & $\mathrm{IM}$ \\
\hline
\end{tabular}




\begin{tabular}{|c|c|c|c|c|}
\hline-0.54 & 1.48 & $\operatorname{tru} B$ & tRNA pseudouridine synthase B & - \\
\hline-0.50 & 1.48 & $\operatorname{purN}$ & Phosphoribosylglycinamide formyltransferase & - \\
\hline-0.48 & 1.32 & $\operatorname{rlu} B$ & Ribosomal large subunit pseudouridine synthase B & - \\
\hline-0.44 & 1.50 & $a c c A$ & $\begin{array}{l}\text { Acetyl-coenzyme A carboxylase carboxyl transferase subunit } \\
\text { alpha }\end{array}$ & Cyto \\
\hline-0.44 & 1.62 & $y c b X$ & Uncharacterized protein $\mathrm{YcbX}$ & - \\
\hline-0.43 & 1.39 & $\operatorname{thr} S$ & Threonine--tRNA ligase & Cyto \\
\hline-0.43 & 1.45 & thiI & tRNA sulfurtransferase & Cyto \\
\hline-0.41 & 1.48 & gltA & Citrate synthase & - \\
\hline-0.41 & 1.45 & $\operatorname{sod} B$ & Superoxide dismutase $[\mathrm{Fe}]$ & - \\
\hline-0.40 & 1.78 & hrpA & ATP-dependent RNA helicase HrpA & - \\
\hline-0.40 & 1.48 & rne & Ribonuclease E & Cyto \\
\hline-0.39 & 2.19 & рерP & Xaa-Pro aminopeptidase & Cyto \\
\hline-0.39 & 1.51 & $\operatorname{ser} C$ & Phosphoserine aminotransferase & Cyto \\
\hline-0.39 & 1.71 & $y b e D$ & UPF0250 protein YbeD & - \\
\hline-0.39 & 1.64 & selB & Selenocysteine-specific elongation factor & Cyto \\
\hline-0.38 & 1.44 & $\operatorname{deg} P$ & Periplasmic serine endoprotease DegP & $\mathrm{IM}$ \\
\hline-0.35 & 1.41 & $\operatorname{teh} B$ & Tellurite methyltransferase & Cyto \\
\hline-0.35 & 1.38 & $\operatorname{gmh} A$ & Phosphoheptose isomerase & Cyto \\
\hline-0.34 & 1.70 & oppF & Oligopeptide transport ATP-binding protein OppF & $\mathrm{IM}$ \\
\hline-0.33 & 1.42 & $u v r A$ & UvrABC system protein A & Cyto \\
\hline-0.31 & 1.34 & $r l m J$ & Ribosomal RNA large subunit methyltransferase J & - \\
\hline-0.30 & 1.38 & $\operatorname{clp} P$ & ATP-dependent Clp protease proteolytic subunit & Cyto \\
\hline-0.30 & 2.52 & helD & Helicase IV & - \\
\hline-0.29 & 1.38 & $\operatorname{csp} B$ & Cold shock-like protein CspB & Cyto \\
\hline-0.29 & 1.69 & fadD & Long-chain-fatty-acid--CoA ligase & $\mathrm{IM}$ \\
\hline-0.27 & 1.42 & $y b a Y$ & Uncharacterized lipoprotein YbaY & $\mathrm{IM}$ \\
\hline-0.24 & 1.55 & yciO & Uncharacterized protein $\mathrm{YciO}$ & - \\
\hline-0.23 & 1.64 & $\operatorname{lrp}$ & Leucine-responsive regulatory protein & - \\
\hline-0.22 & 1.42 & $p n c B$ & Nicotinate phosphoribosyltransferase & - \\
\hline-0.21 & 1.49 & $f d h E$ & Protein FdhE & Cyto \\
\hline-0.21 & 1.35 & $a h p F$ & Alkyl hydroperoxide reductase subunit F & - \\
\hline
\end{tabular}




\section{Figure legends}

\section{Figure 1. The RseP-dependent changes of the proteomic profile of the $E$. coli membrane}

fraction. The identified proteins were ranked in a volcano plot according to their statistical $p$-value (- $\log _{10} p$-value, $y$-axis) and their relative abundance ratios ( $\log _{2}$ fold-change, $x$-axis) between membrane fraction from the wild-type RseP(WT) or a protease active site mutant RseP(E23Q) expressing strains. KK377 ( $\Delta r s e A$ arseP) cells carrying pKK47 (RseP(WT)) or pYK2 (RseP(E23Q)) were grown at $30^{\circ} \mathrm{C}$ in $\mathrm{L}$ medium containing $1 \mathrm{mM}$ IPTG and $1 \mathrm{mM}$ cAMP until mid-log phase. The membrane fractions were prepared by sonical disruptions of cells followed by ultracentrifugation and suspended in PTS solution. After reduction, extracted proteins were digested and the resultant peptides were subjected to nanoLC/MS/MS analyses. The proteins with $p$-value $<0.05$ and $\log _{2}$ fold-change $<0$ are indicated by red cicles, and $p$-value $<0.05$ and $\log _{2}$ fold-change $>0$ are indicated by blue circles, and the other proteins are indicated by black circles.

\section{Figure 2. The proteolytic function of RseP is necessary for the ferric citrate-dependent} transcriptional activation of the $f e c$ operon. (A) A schematic representation of the Fec system. Ferric citrate is transported by outer membrane transporter FecA into the periplasm and then iron is transported into the cytoplasm by periplasmic protein FecB and $\mathrm{ABC}$ transporter FecCDE. FecR, a type-II ( $\left.\mathrm{N}_{\text {in }}-\mathrm{C}_{\text {out }}\right)$ single-pass inner membrane protein, receives the signal of ferric citrate from FecA and transmits it to FecI, an alternative sigma factor. Activated FecI induces the transcription of the $f e c A B C D E$ operon ( $f e c$ operon). The expression of the $f e c I R$ operon encoding FecI and FecR is independent of the FecI activity. OM, Peri, IM, and, Cyto indicate outer membrane, periplasm, inner membrane, and cytoplasm, respectively. (B) RseP dependency of the $\mathrm{P}_{\text {fecA-lac }}$ reporter expression in response to ferric citrate. YH426 $\left(\Delta o m p A \Delta o m p C r s e P^{+}\right)$or KA363 $(\Delta o m p A \Delta o m p C$ 


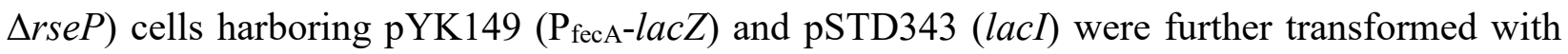
pSTD1060 (vec), pYK151 (RseP(WT)-HM, WT) or pYK153 (RseP(E23Q)-HM). The cells were inoculated into M9-based medium containing $1 \mathrm{mM}$ IPTG, $1 \mathrm{mM}$ cAMP, and $0.1 \mu \mathrm{M} \mathrm{FeCl}{ }_{3}$ supplemented with or without $1 \mathrm{mM} \mathrm{Na} 3$-citrate, grown at $30^{\circ} \mathrm{C}$ until mid-log phase and their LacZ activities were measured. The relative LacZ activities normalized by the LacZ activity of the $r s e P^{+}$ cells grown in medium containing $1 \mathrm{mM} \mathrm{Na}$-citrate are shown (see Experimental procedures for details). Two independent experiments were carried out and the mean values are shown with standard deviations. (C) Cellular accumulation of the RseP proteins. The cells were grown as in (B) and the proteins were analyzed by $12.5 \%$ Laemmli SDS-PAGE and anti-RseP ( $\alpha$-RseP) or antiSecB $(\alpha-\operatorname{SecB})$ immunoblotting. RseP-HM shown on the right indicates RseP(WT)-HM or RseP(E23Q)-HM expressed from pKK47 (RseP(WT)) or pYK2 (RseP(E23Q), and RseP indicates the chromosomally-encoded RseP. The asterisk indicates a possible degradation product of RseP(WT)-HM or RseP(E23Q)-HM. Cytoplasmic protein SecB serves as a loading control. The positions of molecular size markers (in $\mathrm{kDa}$ ) are shown on the left. Note that the band intensity of RseP-HM is saturated.

Figure 3. RseP is involved in the proteolytic processing of FecR. (A) Schematic representations of FecR and the N-terminally 3xFLAG-tagged FecR model substrate (F-FecR). The predicted transmembrane region and the $3 \times$ FLAG tag are shown as the black and the white boxes, respectively. FL, full length F-FecR. (B) Ability of various FecR model substrates to transmit the ferric citrate signal. MC4100 $\left(f e c R^{+}\right)$or YK627 $(\Delta f e c R)$ cells harboring pYK149 $\left(\mathrm{P}_{\text {fecA-lacZ }}\right)$ and pSTD343 (lacl) were further transformed with pSTD1060 (vec), pYK186 (pFecR), pYK188 (pFFecR) or pYK190 (pF-MBP-FecR). The cells were grown at $30^{\circ} \mathrm{C}$ in M9-based medium containing $1 \mathrm{mM}$ IPTG, $1 \mathrm{mM}$ cAMP, and $0.1 \mu \mathrm{M} \mathrm{FeCl}_{3}$ supplemented with or without $1 \mathrm{mM} \mathrm{Na}$-citrate, and 
the LacZ activity was measured as in Fig. 2B. The relative LacZ activities normalized by the LacZ activity of $f e c R^{+}$cells grown in medium containing $1 \mathrm{mM} \mathrm{Na}$-citrate are shown. (C) Behaviors of the F-FecR protein in the presence or absence of the co-expression of $\mathrm{RseP}$ and the addition of $\mathrm{Na}_{3}$ citrate. YK167 ( $\left.\Delta r s e A, r s e P^{+}\right)$or YK191 ( $\left.\Delta r s e A \Delta r s e P, \Delta r s e P\right)$ cells harboring pTWV228 (vec) or pYK130 (pF-FecR) were further transformed with pSTD689 (vec), pYH9 (RseP-HM, WT) or pYH13 (RseP E23Q-HM, E23Q). The cells were grown at $30^{\circ} \mathrm{C}$ in M9-based medium containing $1 \mathrm{mM}$ IPTG, $1 \mathrm{mM}$ cAMP, $10 \mu \mathrm{M} \mathrm{FeCl}_{3}$ supplemented with or without $1 \mathrm{mM} \mathrm{Na}$-citrate and grown as in Fig. 2B. The proteins were analyzed by $15 \%$ Bis-Tris SDS-PAGE and anti-FLAG $(\alpha-$ FLAG), or $12.5 \%$ Laemmli SDS-PAGE and anti-RseP ( $\alpha$-RseP), or anti-SecB $(\alpha-\operatorname{SecB})$ immunoblotting. FL, CL(a), CL(b), and CL(c) on the right indicate full-length F-FecR, the possible cleavage product (a), (b), and (c), respectively. Note that the band intensities of F-FecR FL, F-FecR CL(a) and RseP-HM are saturated.

Figure 4. RseP cleaves the transmembrane region of FecR. (A) Schematic representations of FecR, the F-MBP-FecR model substrate, and the truncated derivatives of F-MBP-FecR. The regions corresponding to the FecR-derived cytoplasmic and periplasmic domains are shown as white lines, MBP as the gray line, the predicted transmembrane region as a black box, and 3xFLAG tag as a white box, respectively. (B) Behavior of the F-MBP-FecR protein in response to the coexpression of RseP and the addition of $\mathrm{Na}_{3}$-citrate. YK167 $\left(\Delta r s e A, r s e P^{+}\right)$or YK191 $(\Delta r s e A \Delta r s e P)$ cells harboring pTWV228 (vec) or pYK147 (pF-MBP-FecR) were further transformed with pSTD689 (vec), pYH9 (RseP-HM, WT) or pYH13 (RseP(E23Q)-HM, E23Q). The cells were grown as in Fig. 3C. The proteins were analyzed by 10\% Laemmli SDS-PAGE and anti-FLAG immunoblotting or $12.5 \%$ Laemmli SDS-PAGE and anti-RseP $(\alpha-\mathrm{RseP})$, or anti-SecB $(\alpha-\mathrm{SecB})$ immunoblotting. The band intensity of RseP-HM is saturated. (C) Behaviors of the truncated 
derivatives of F-MBP-FecR. YK191 cells harboring pSTD689 (vec), pYH9 (WT), or pYH13 (E23Q) were further transformed with pYK147 derivatives encoding the truncated mutant forms of F-MBP-FecR shown in (A). The cells were grown and analyzed as in (B). The lanes with an asterisk were the same sample used for the comparison of the band sizes. Note that the intensities of RseP-HM and some of F-MBP-FecR and its derivative CL(a), CL(b) and CL(c) bands are saturated.

\section{Figure 5. Kinetics and RseP-dependence of the generation of the FecR-derived fragments.}

(A) Pulse-chase analysis of the F-MBP-FecR cleavage by RseP. YK191 ( $\Delta r s e A \Delta r s e P$ ) cells harboring pTWV228 (vec) or pYK147 (pF-MBP-FecR) were further transformed with pSTD689 (vec), pYH9 (RseP-HM, WT), or pYH13 (RseP(E23Q)-HM, E23Q). The cells were grown at $30^{\circ} \mathrm{C}$ in M9-based medium containing $10 \mu \mathrm{M} \mathrm{FeCl}_{3}, 1 \mathrm{mM} \mathrm{Na}$-citrate until early-log phase. After induction with $1 \mathrm{mM}$ IPTG and $1 \mathrm{mM}$ cAMP for $10 \mathrm{~min}$, proteins were pulse-labeled with $\left[{ }^{35} \mathrm{~S}\right]$ methionine for $1 \mathrm{~min}$ followed by chase with an excess amount of unlabeled methionine for the indicated periods. The proteins were analyzed by anti-FLAG immunoprecipitation, 10\% Laemmli SDS-PAGE, and phosphorimaging. Asterisk indicates a non-specific background band. (B) Quantification of the experimental results shown in (A). The percentage of the intensity of each F-MBP-FecR fragment in total band intensities was plotted versus the chase time. The averages of the values from two independent experiments are shown with standard deviations. Because the exact number of the Met residue in the cleaved fragments is not known, the band intensities were not corrected by the number of Met.

Figure 6. RseP-dependent conversion of the FecR CL(b) fragment to CL(c). (A) Pulse-chase analysis of the RseP-dependent cleavage of F-MBP-FecR. YK191 ( $\Delta r s e A \Delta r s e P$ ) cells harboring 
pYH604 (constitutive expression vector pTWV228c, vec) or pYK182 (pF-MBP-FecR) were further transformed with pSTD689 (vec), pYH9 (RseP-HM, WT) or pYH13 (RseP E23Q-HM, E23Q). The cells were grown and analyzed as in Fig. 5A except that F-MBP-FecR was expressed constitutively and that RseP was induced after the start of the chase. Following pulse-labelling with $\left[{ }^{35} \mathrm{~S}\right]$ methionine for $1 \mathrm{~min}$, chase was initiated by the addition of unlabeled methionine. The RseP proteins were induced with 1.2 $\mathrm{mM}$ IPTG and $1.2 \mathrm{mM}$ cAMP $5.5 \mathrm{~min}$ after the initiation of the case (indicated as $0 \mathrm{~min}$ ). The timing of the chase-start and the RseP induction was shown under the gel image. (B) Quantification of the results of the experiments shown in (A). The percentage of each band intensity to the total band intensity are plotted. (C) Behaviors of the truncated derivatives of F-MBP-FecR in response to the proteolytic activity of RseP. YK191 cells harboring pSTD689 (vec), pYH9 (WT) or pYH13 (E23Q) were further transformed with a plasmid encoding the F-MBP-FecR derivatives as in Fig. 4A. The cells were grown and analyzed as in Fig. 4B.

\section{Figure 7. The $\mathrm{CL}(\mathrm{c})$ fragment can activate the expression of the fec operon in an RseP-} independent manner. (A) The behaviors of F-FecR and F-FecR85 in response to the addition of $\mathrm{Na}_{3}$-citrate. MC4100 $\left(f e c R^{+}\right)$or YK627 ( $\left.\Delta f e c R\right)$ cells harboring pYK149 ( $\left.\mathrm{P}_{\text {fecA-lacZ }}\right)$ and pSTD343 (lacl) were further transformed with pSTD1060 (vec), pYK186 (pFecR, FL), pYK198 (pFecR85), pYK188 (pF-FecR, FL), or pYK200 (pF-FecR85). The cells were grown as in Fig. 2B and proteins were analyzed as in Fig. 3C. Note that the band intensities of F-FecR FL and CL(a), and F-FecR85 are saturated. (B) The citrate-dependency of the fec operon activations mediated by the FecR and F-FecR derivatives. The cells are grown and the LacZ activity was measured as in Fig. 3B. (C) The RseP-dependency of the $f e c$ operon activations mediated by F-FecR85. YK630 ( $\left.r s e P^{+}\right)$or YK797

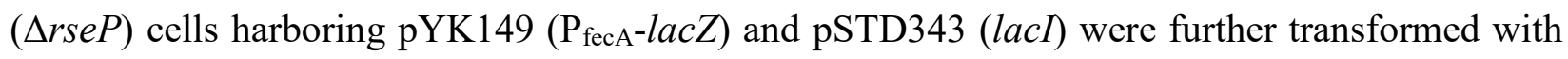
pYK188 (pF-FecR, FL) or pYK200 (pF-FecR85). The cells were grown and the LacZ activity was 
measured as in (B). The relative LacZ activities were normalized by the LacZ activity of the cells expressing F-FecR that had been grown in medium containing $1 \mathrm{mM} \mathrm{Na}$-citrate are shown.

Figure 8. A model of the sequential processing of FecR and the involvement of RseP in it in the ferric citrate-induced signal transduction. (i) FecR undergoes a self-cleavage immediately after translation to generate $\mathrm{CL}(\mathrm{a})$. (ii) $\mathrm{CL}(\mathrm{a})$ is cleaved or trimmed by unknown protease(s) in the periplasm to yield CL(b), which is promoted by the ferric citrate signal. (iii) CL(b) then receives RseP-mediate intramembrane proteolysis inside the membrane to release CL(c) from the membrane. (iv) sigma factor FecI bound to CL(c) activates the transcription of the fec operon. 
bioRxiv preprint doi: https://doi.org/10.1101/2021.02.04.429786; this version posted February 4, 2021. The copyright holder for this preprint (which was not certified by peer review) is the author/funder, who has granted bioRxiv a license to display the preprint in perpetuity. It is made available under aCC-BY 4.0 International license.

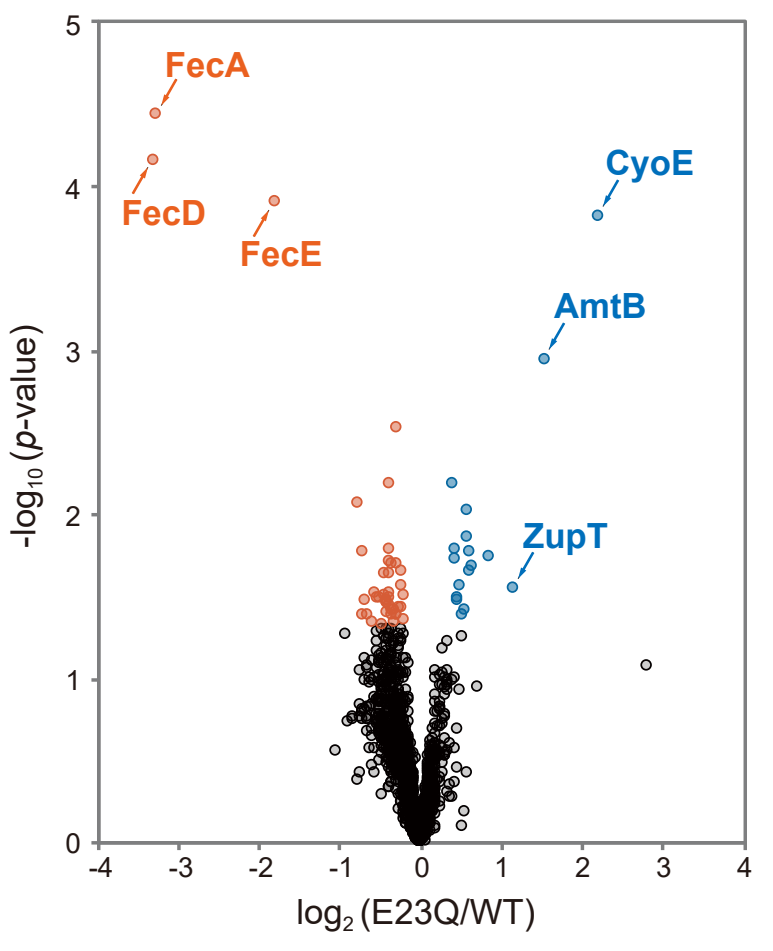

\section{Yokoyama et al., Figure 1}


A

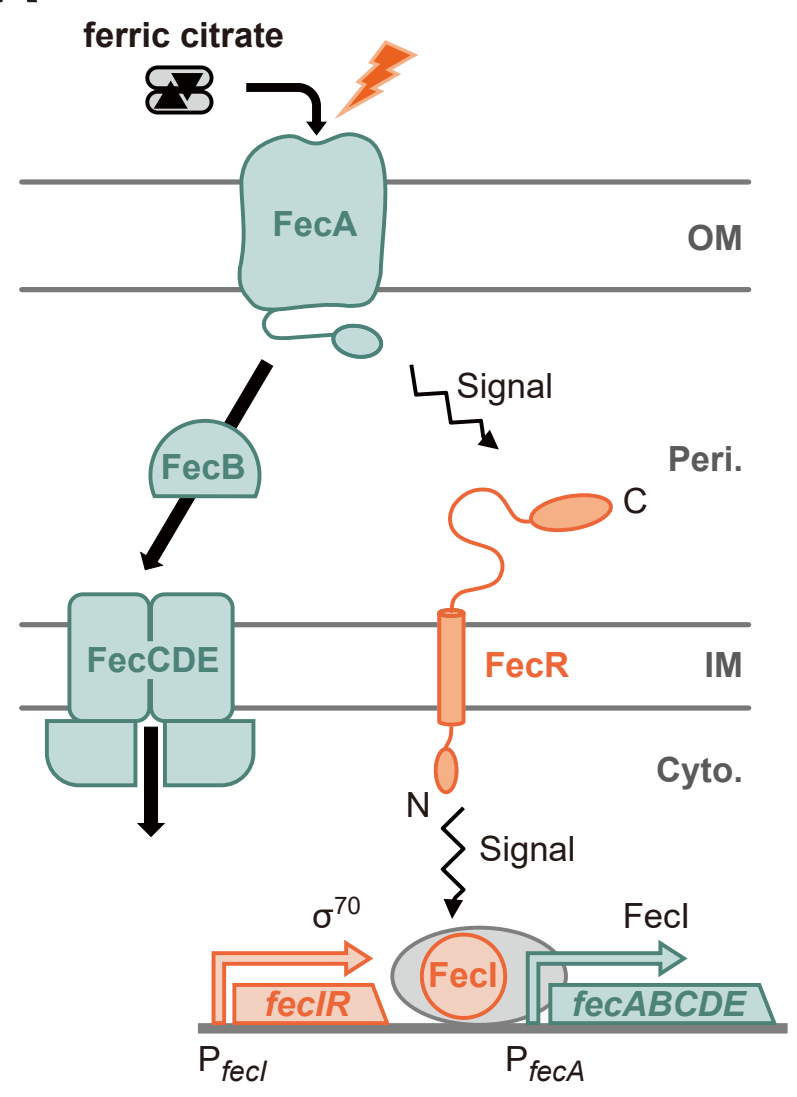

B

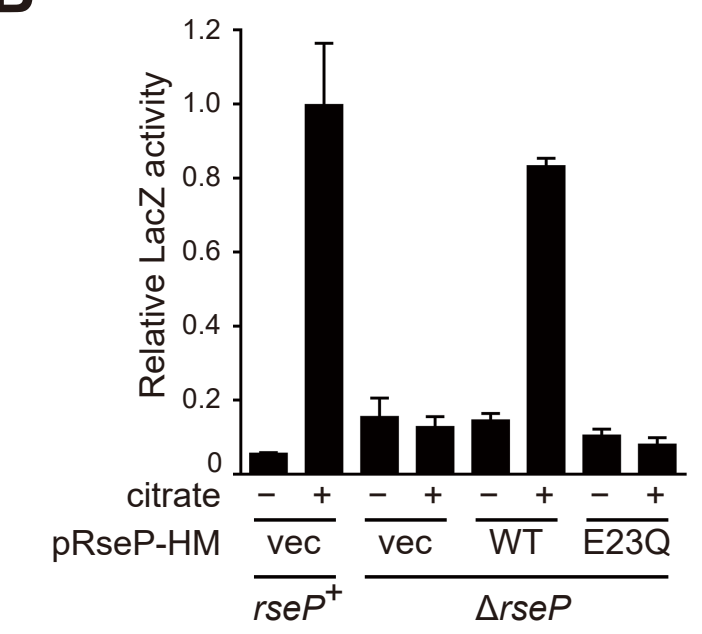

C

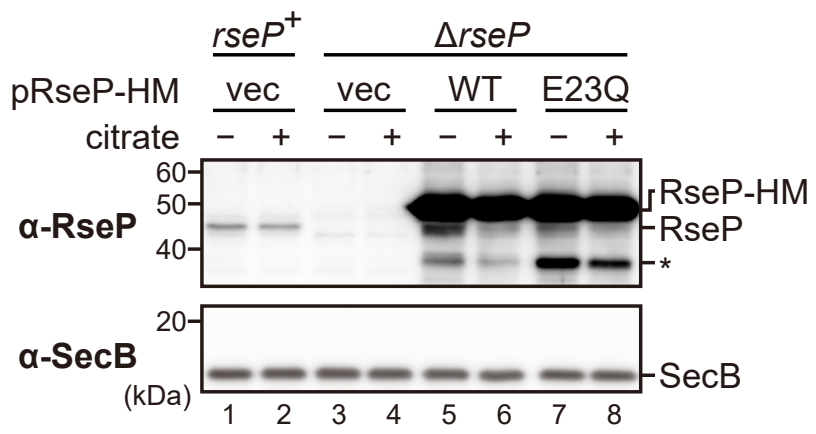

\section{Yokoyama et al., Figure 2}




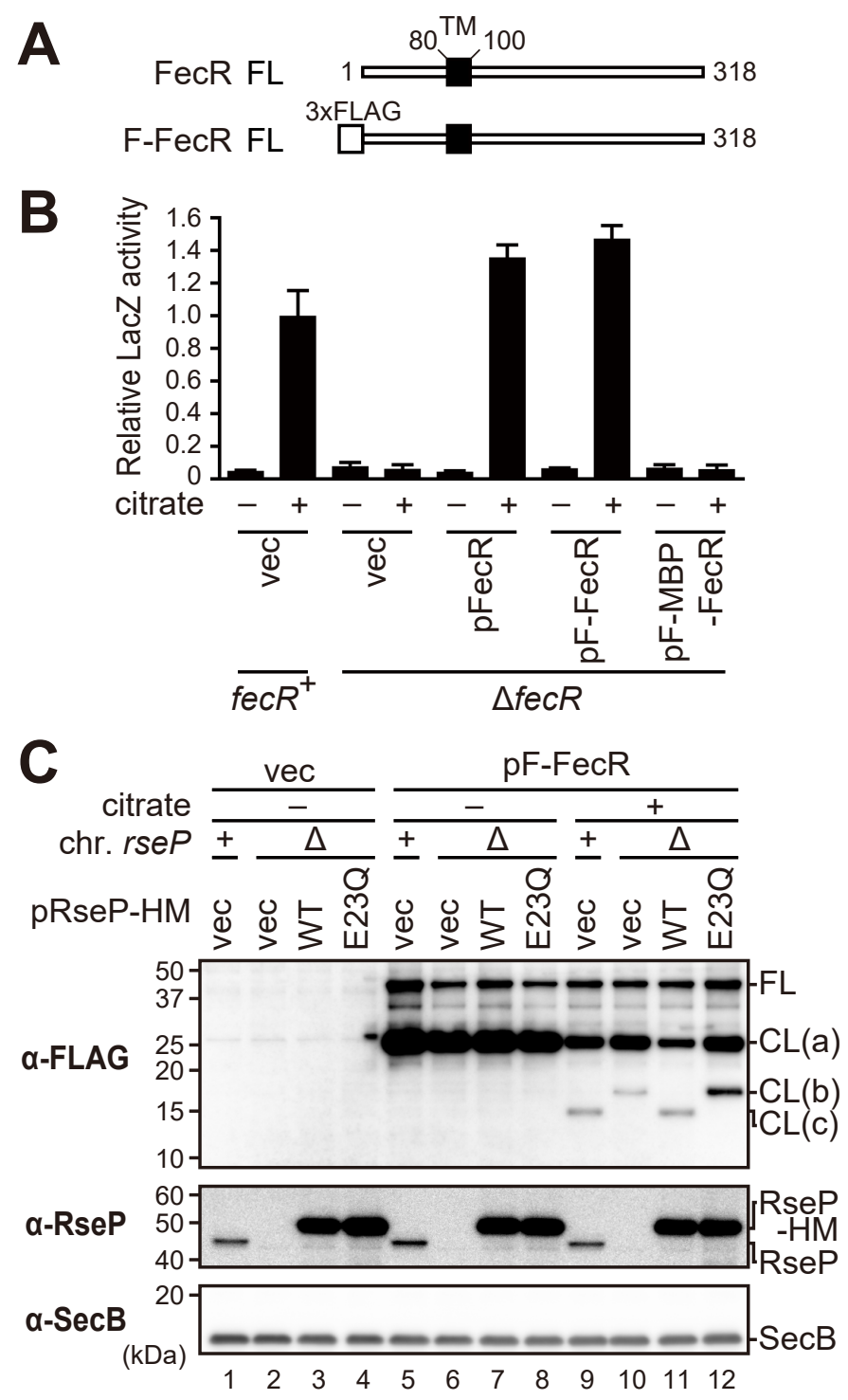

\section{Yokoyama et al., Figure 3}


A

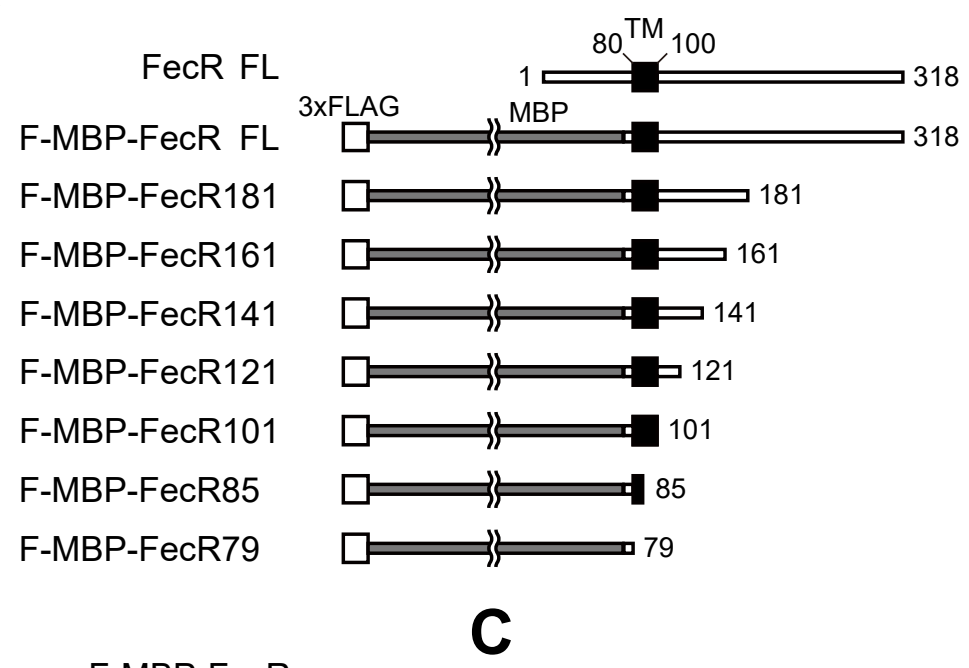

B
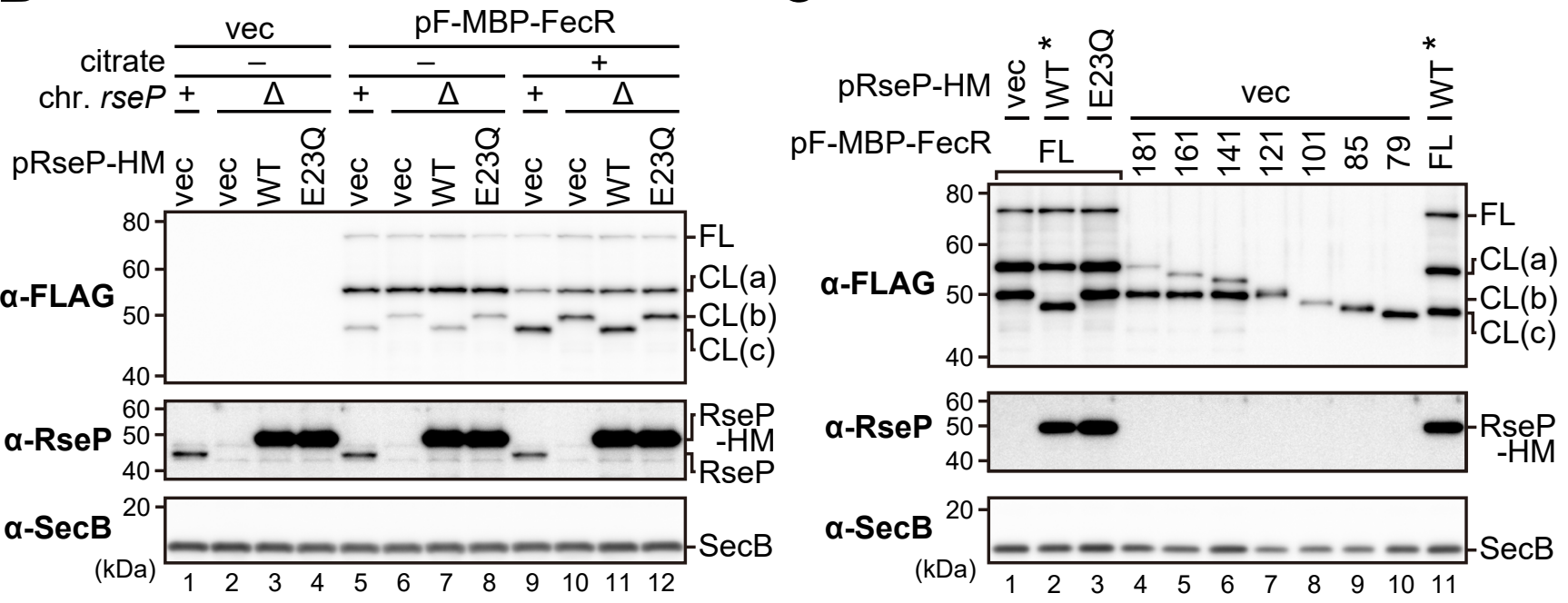


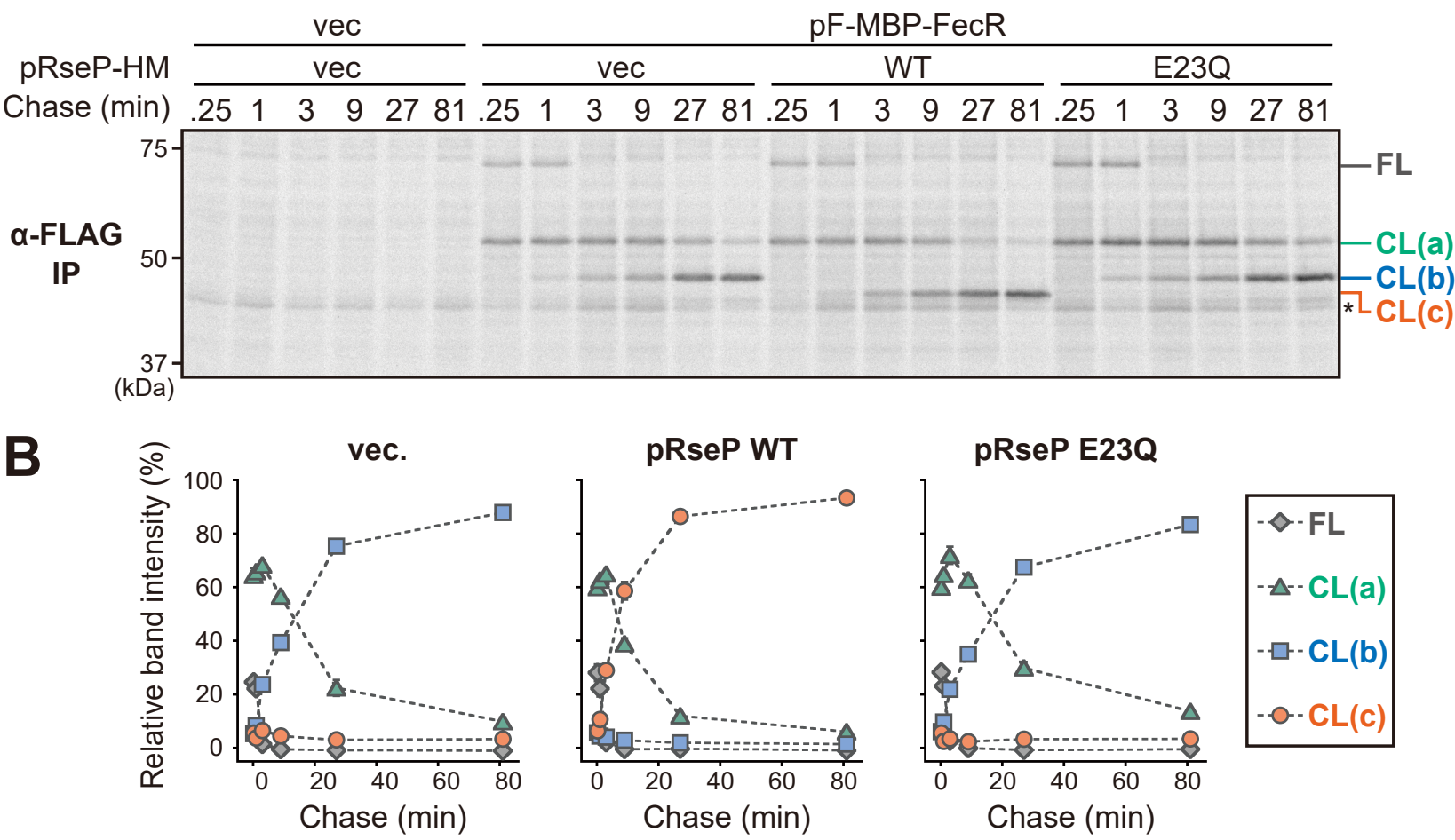

\section{Yokoyama et al., Figure 5}




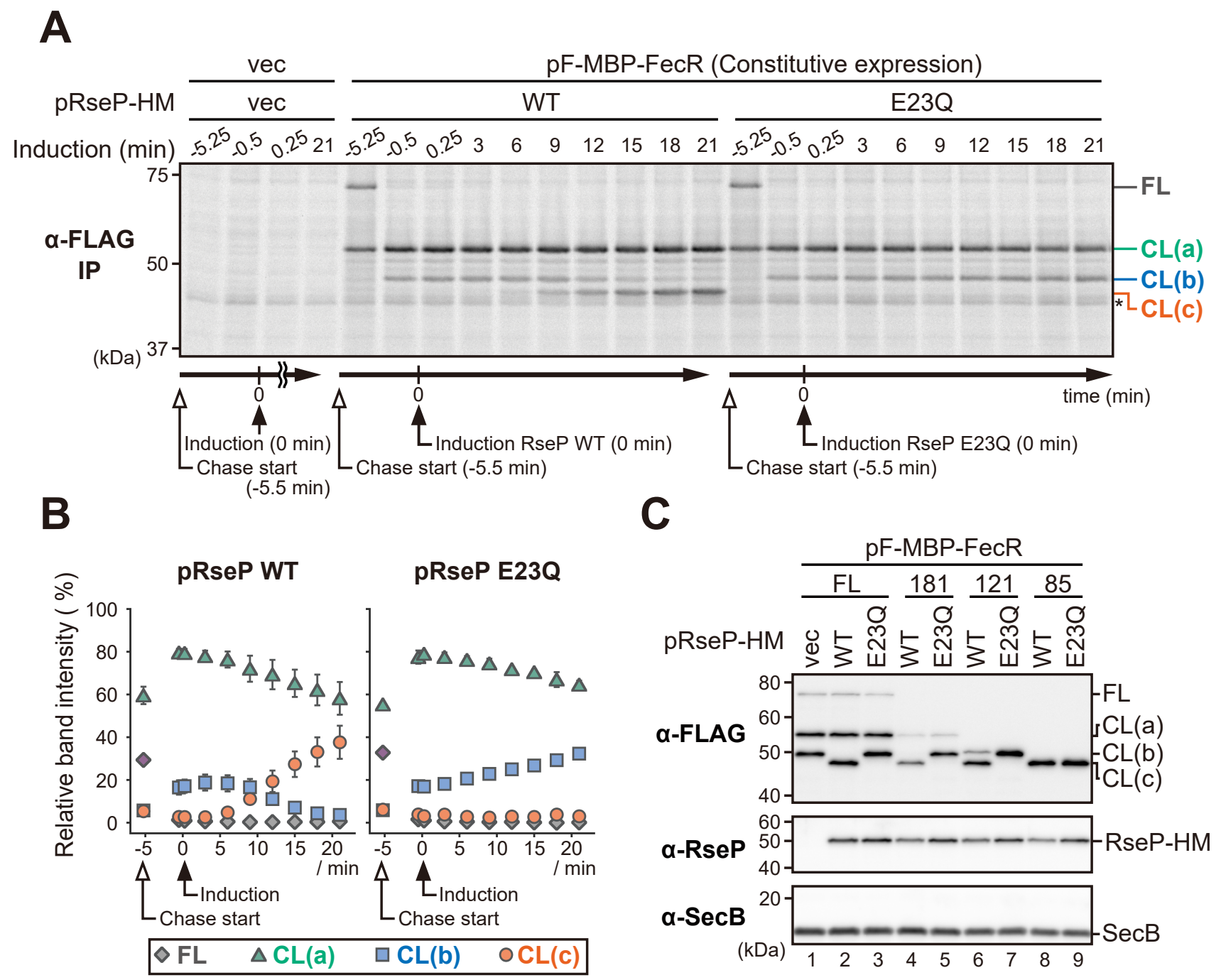

\section{Yokoyama et al., Figure 6}


A

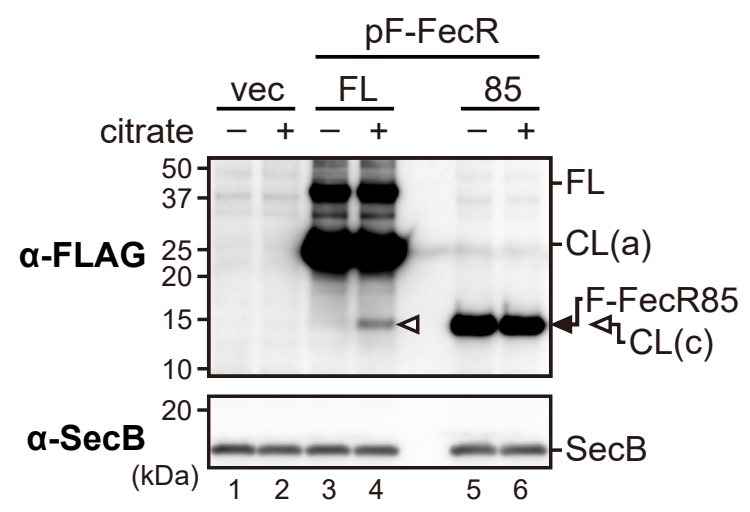

B

C

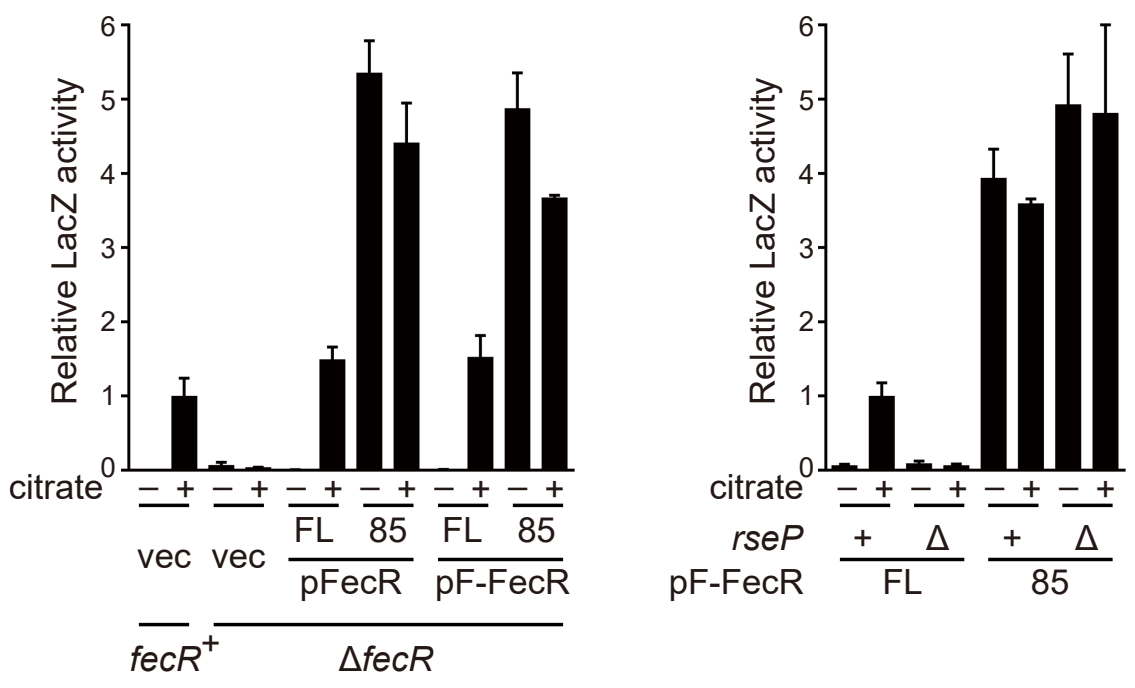

Yokoyama et al., Figure 7 
bioRxiv preprint doi: https://doi org/10.1101/2021.02 04.429786. this version posted February 4, 2021. The copyright holder for this preprint (which was not certified by peer review) is the author/funder, who has granted bioRxiv a license to display the preprint in perpetuity. It is made available under aCC-BY 4.0 International license.

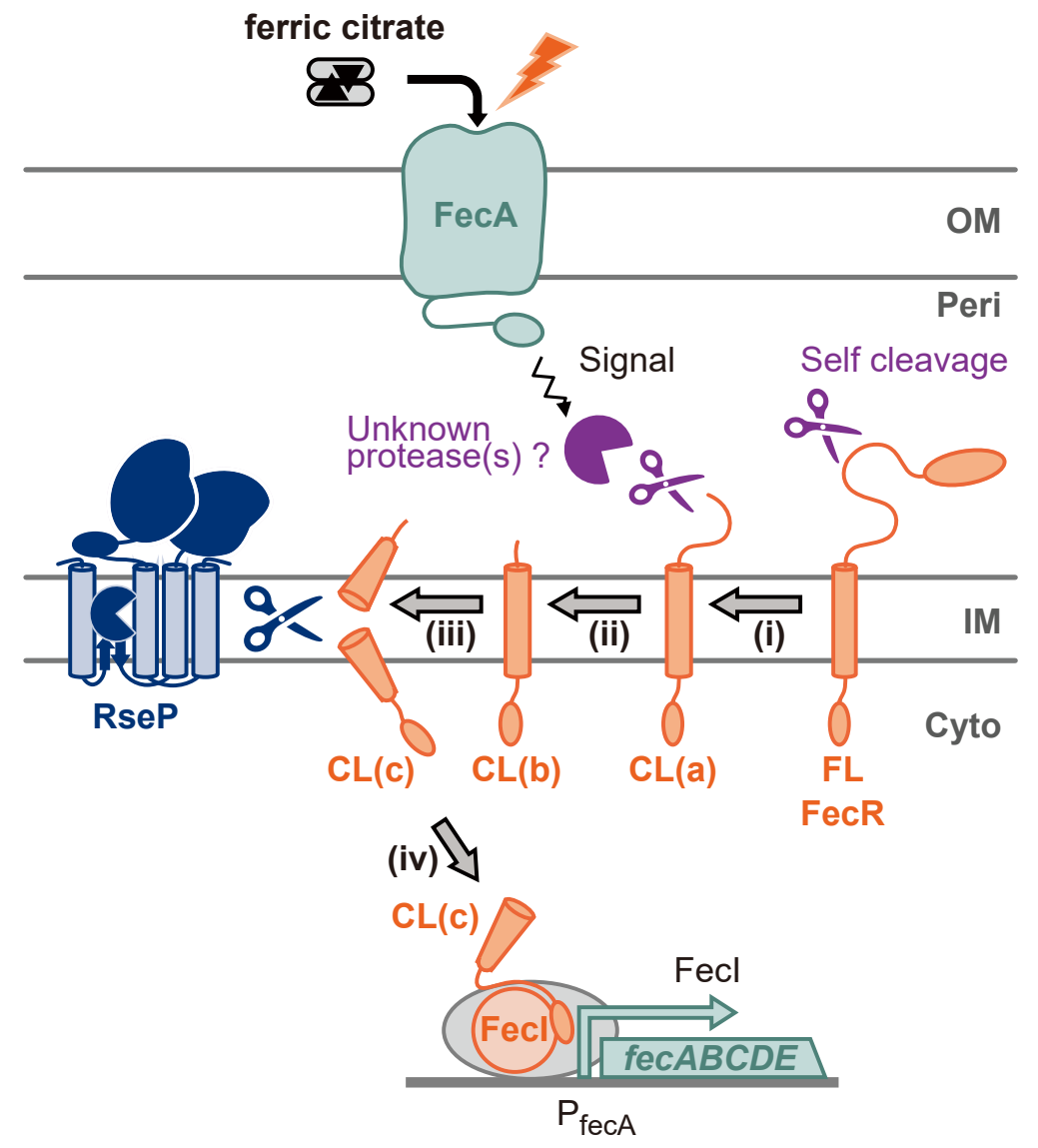

Yokoyama et al., Figure 8 